\title{
OPTIMALITY CONDITIONS AND ERROR ANALYSIS OF SEMILINEAR ELLIPTIC CONTROL PROBLEMS WITH $L^{1}$ COST FUNCTIONAL*
}

\author{
EDUARDO CASAS ${ }^{\dagger}$, ROLAND HERZOG $^{\ddagger}$, AND GERD WACHSMUTH ${ }^{\ddagger}$
}

\begin{abstract}
Semilinear elliptic optimal control problems involving the $L^{1}$ norm of the control in the objective are considered. Necessary and sufficient second-order optimality conditions are derived. A priori finite element error estimates for piecewise constant discretizations for the control and piecewise linear discretizations of the state are shown. Error estimates for the variational discretization of the problem in the sense of [M. Hinze, Comput. Optim. Appl., 30 (2005), pp. 45-61] are also obtained. Numerical experiments confirm the convergence rates.
\end{abstract}

Key words. optimal control of partial differential equations, nondifferentiable objective, sparse controls, finite element discretization, a priori error estimates

AMS subject classifications. 35J61, 49K20, 49M25

DOI. $10.1137 / 110834366$

1. Introduction. In this paper we consider an optimal control problem subject to a semilinear elliptic state equation. The objective functional contains the $L^{1}$ norm of the control and it is therefore nondifferentiable. Problems of this type are of interest for two reasons. First, the $L^{1}$ norm of the control is often a natural measure of the control cost. Second, this term leads to sparsely supported optimal controls, which are desirable, for instance, in actuator placement problems [17]. In optimal control of distributed parameter systems, it may be impossible or undesirable to put the controllers at every point of the domain. Instead, we can decide to control the system by localizing the controls in small regions. The big issue is to determine the most effective location of the controls. An answer to this question is given by solving the control problem with an $L^{1}$ norm of the control.

However, the nondifferentiability of the objective leads to some difficulties. While first-order necessary optimality conditions can be derived in a standard way via Clarke's calculus of generalized derivatives, second-order conditions require additional effort. From the first-order optimality conditions, we deduce a representation formula (see (3.5c)) for the subdifferential $\bar{\lambda}$ of the nondifferentiable term at the optimal control $\bar{u}$, i.e., $\bar{\lambda} \in \partial\|\bar{u}\|_{L^{1}(\Omega)}$. This formula is new and it has some important consequences. First, it proves the uniqueness of $\bar{\lambda}$, which is not usually obtained for a nondifferentiable optimization problem. Second, it proves that $\bar{\lambda}$ is not only an $L^{\infty}(\Omega)$ function, but it is a Lipschitz function in $\bar{\Omega}$, which implies, with formula (3.5a) for the optimal control, that $\bar{u}$ is also Lipschitz in $\bar{\Omega}$. This extra regularity for the optimal control is essential in deriving the error estimates. We should emphasize that there are no error estimates if we do not have extra regularity of the optimal control. Moreover,

*Received by the editors May 17, 2011; accepted for publication (in revised form) March 16, 2012; published electronically July 12, 2012.

http://www.siam.org/journals/siopt/22-3/83436.html

${ }_{\dagger}^{\dagger}$ Departmento de Matemática Aplicada y Ciencias de la Computación, E.T.S.I. Industriales y de Telecomunicación, Universidad de Cantabria, 39005 Santander, Spain (eduardo.casas@unican.es). This author was partially supported by the Spanish Ministerio de Economía y Competitividad under the project MTM2011-22711.

${ }^{\ddagger}$ Faculty of Mathematics, Chemnitz University of Technology, 09126 Chemnitz, Germany (roland.herzog@mathematik.tu-chemnitz.de, gerd.wachsmuth@mathematik.tu-chemnitz.de).

795 
the representation formulas (3.5a) and (3.5c), along with their discrete counterparts, allow us to derive $L^{\infty}$ error estimates for $\bar{u}-\bar{u}_{h}$ and $\bar{\lambda}-\bar{\lambda}_{h}$, which is important for this problem because it shows that we can identify in a precise way the region where the optimal control is vanishing by solving the discrete control problem.

An important part of the paper is devoted to the second-order optimality conditions. Since the partial differential equation is not linear, the control problem is not convex in general; therefore, we have to determine second-order conditions to deal with local minima. It is well known that the sufficient second-order condition is a crucial tool in proving error estimates. In the second-order analysis, due to the nonsmoothness of the problem, the most delicate point was to discover the correct cone of critical directions $C_{\bar{u}}$; see (3.6). We found the correct one, allowing us to prove both second-order necessary and sufficient optimality conditions with the minimal gap.

The second-order sufficient conditions are subsequently put to use to derive a priori error estimates for a finite element discretization of the problem at hand. We employ piecewise linear and continuous elements for the state and adjoint state and piecewise constants for the control. We obtain an estimate of order $h$ w.r.t. the $L^{\infty}$ norm for the control, state, and adjoint state. The proof of these estimates requires a deeper analysis than the one used in smooth optimal control problems, and it is not a straightforward extension of the techniques established for smooth problems. The proof exploits the $W^{2, p}$ regularity of the optimal adjoint state, and the consequential Lipschitz regularity of the optimal control, which hold under the assumption of a smooth boundary of the domain $\Omega$. Since smooth domains cannot be triangulated exactly, the error estimate takes into account the additional error from replacing $\Omega$ by a polygonal approximation $\Omega_{h}$. As mentioned in Remark 2.4, all results also remain valid for a convex polygonal domain of $\mathbb{R}^{2}$. Numerical experiments verify the theoretical convergence order for both cases, smooth and convex polygonal domains. Finally, we derive error estimates for a variational discretization of the control problem. More precisely, we discretize the states and the state equation, but there is no discretization of the control. This procedure suggested by Hinze [13] leads to optimal error estimates (order $h^{2}$ ), and the discrete problem can still be solved numerically.

Let us put this work into perspective. A problem with an $L^{1}$ term in the objective was analyzed in [17], subject to a linear elliptic equation. Second-order conditions are not required there since this problem is convex. A priori and a posteriori error estimates for this case were provided in [19]. The authors in [9] analyze algorithms for optimal control problems which involve the norm of a nonreflexive control Banach space.

This paper is organized as follows. We present the problem setting and some preliminary results in section 2 . Section 3 is devoted to the development of firstand second-order necessary and sufficient optimality conditions. We study the finite element error for the case of full discretization in section 4 and in section 5 for the case of variational discretization. We report on numerical experiments which confirm our results in section 6 .

2. Setting of the problem and preliminary results. In this paper, $\Omega$ will denote an open bounded subset of $\mathbb{R}^{n}, n=2$ or 3 , with a $C^{1,1}$ boundary $\Gamma$. In this domain we consider the following control problem:

$$
\left\{\begin{array}{l}
\min J(u), \\
\alpha \leq u(x) \leq \beta \text { for a.a. } x \in \Omega,
\end{array}\right.
$$


where $J(u)=F(u)+\mu j(u)$, with $F: L^{2}(\Omega) \longrightarrow \mathbb{R}$ and $j: L^{1}(\Omega) \longrightarrow \mathbb{R}$ defined by

$$
F(u)=\int_{\Omega} L\left(x, y_{u}(x)\right) \mathrm{d} x+\frac{\nu}{2} \int_{\Omega} u^{2}(x) \mathrm{d} x \text { and } j(u)=\int_{\Omega}|u(x)| \mathrm{d} x,
$$

$y_{u}$ being the solution of the state equation

$$
\left\{\begin{aligned}
A y+a(x, y) & =u & & \text { in } \Omega, \\
y & =0 & & \text { on } \Gamma .
\end{aligned}\right.
$$

$A$ is the linear operator

$$
A y=-\sum_{i, j=1}^{n} \partial_{x_{j}}\left[a_{i j}(x) \partial_{x_{i}} y\right]+a_{0}(x) y .
$$

We make the following assumptions on the functions and parameters involved in the control problem (P).

Assumption 1. The coefficients of $A$ have the following regularity properties: $a_{0} \in L^{\infty}(\Omega), a_{i j} \in C^{0,1}(\bar{\Omega})$, and

$$
a_{0}(x) \geq 0 \text { and } \sum_{i, j=1}^{n} a_{i j}(x) \xi_{i} \xi_{j} \geq \Lambda|\xi|^{2} \text { for a.a. } x \in \Omega \text { and } \forall \xi \in \mathbb{R}^{n} \text {. }
$$

Assumption 2. $a: \Omega \times \mathbb{R} \longrightarrow \mathbb{R}$ is a Carathéodory function of class $C^{2}$ with respect to the second variable, with $a(\cdot, 0) \in L^{\bar{p}}(\Omega)$ for some $n<\bar{p}$, and satisfying

$$
\left\{\begin{array}{l}
\frac{\partial a}{\partial y}(x, y) \geq 0 \text { for a.a. } x \in \Omega \text { and } \forall y \in \mathbb{R}, \\
\forall M>0 \exists C_{M}>0 \text { s.t. } \sum_{j=1}^{2}\left|\frac{\partial^{j} a}{\partial y^{j}}(x, y)\right| \leq C_{M} \text { for a.a. } x \in \Omega \text { and }|y| \leq M .
\end{array}\right.
$$

Assumption 3. We also assume $-\infty<\alpha<0<\beta<+\infty, \mu>0, \nu>0$, and $L: \Omega \times \mathbb{R} \longrightarrow \mathbb{R}$ is a Carathéodory function of class $C^{2}$ w.r.t. the second variable such that $L(\cdot, 0) \in L^{1}(\Omega)$ and for every $M>0$ there exists a function $\psi_{M} \in L^{\bar{p}}(\Omega)$, with $n<\bar{p}<+\infty$, satisfying

$$
\left|\frac{\partial^{j} L}{\partial y^{j}}(x, y)\right| \leq \psi_{M}(x) \quad \forall|y| \leq M \text { and for a.a. } x \in \Omega, \text { with } j=1,2 .
$$

In what follows, we will denote the set of feasible controls by

$$
\mathbb{K}=\left\{u \in L^{\infty}(\Omega): \alpha \leq u(x) \leq \beta \text { for a.a. } x \in \Omega\right\} .
$$

Let us notice that the usual function $L(x, y)=\frac{1}{2}\left(y-y_{d}(x)\right)^{2}$ satisfies Assumption 3 if $y_{d} \in L^{\bar{p}}(\Omega)$.

Remark 2.1. In Assumption 3 we made the hypothesis $\alpha<0<\beta$. In the case where $0 \leq \alpha \leq \beta$ or $\alpha \leq \beta \leq 0$, the $L^{1}$ norm is linear; hence the cost functional $J$ is differentiable, and the control problem (P) falls into the framework of well studied optimal control problems. Here we are interested in analyzing the nondifferentiable case. 
Moreover, since we are looking for sparsity of the optimal control, it does not make sense to consider $0<\alpha$ or $\beta<0$. However, the cases $\alpha=0$ or $\beta=0$ are frequent in practice. In these situations, the sparsity of the optimal control is also induced by the presence of the term $\mu\|u\|_{L^{1}(\Omega)}$; see Remark 3.3.

The next theorem states that the control-to-state map is well posed and differentiable.

THEOREM 2.2. The following statements hold:

1. For any $u \in L^{p}(\Omega)$, with $n / 2<p \leq \bar{p}$, there exists a unique solution of (2.1) $y_{u} \in W^{2, p}(\Omega)$.

2. The mapping $G: L^{p}(\Omega) \longrightarrow W^{2, p}(\Omega)$ defined by $G(u)=y_{u}$ is of class $C^{2}$. Moreover, for $v \in L^{p}(\Omega), z_{v}=G^{\prime}(u) v$ is the unique solution of

$$
\left\{\begin{aligned}
A z+\frac{\partial a}{\partial y}(x, y) z=v & & \text { in } \Omega, \\
z=0 & & \text { on } \Gamma,
\end{aligned}\right.
$$

and given $v_{1}, v_{2} \in L^{p}(\Omega), w_{v_{1}, v_{2}}=G^{\prime \prime}(u)\left(v_{1}, v_{2}\right)$ is the unique solution of



where $z_{v_{i}}=G^{\prime}(u) v_{i}, i=1,2$.

The existence and uniqueness of a solution of $(2.1)$ in $H_{0}^{1}(\Omega) \cap L^{\infty}(\Omega)$ is obtained by classical arguments; see, for instance, [4]. The $W^{2, p}(\Omega)$ regularity follows from the $C^{1,1}$ regularity of $\Gamma$, Assumptions 1 and 2, and the result of Grisvard [11, Theorem 2.4.2.5]. The differentiability of $G$ can be obtained from the implicit function theorem as follows. We define the nonlinear operator

$$
\mathcal{F}:\left[W^{2, p}(\Omega) \cap W_{0}^{1, p}(\Omega)\right] \times L^{p}(\Omega) \longrightarrow L^{p}(\Omega), \quad \mathcal{F}(y, u)=A y+a(\cdot, y)-u .
$$

Then, it is immediate to check that $\mathcal{F}$ is of class $C^{2}$ and $\mathcal{F}(G(u), u)=0$ for every $u \in L^{p}(\Omega)$. Using [11, Theorem 2.4.2.5] again, we deduce that

$$
\frac{\partial \mathcal{F}}{\partial y}(G(u), u): W^{2, p}(\Omega) \cap W_{0}^{1, p}(\Omega) \longrightarrow L^{p}(\Omega)
$$

is an isomorphism. Thus, the assumptions of the implicit function theorem are fulfilled and some simple calculations prove (2.5) and (2.6).

As an immediate consequence of the previous theorem, we get that the smooth part $F$ of the objective functional enjoys the following differentiability result.

Theorem 2.3. Functional $F: L^{2}(\Omega) \longrightarrow \mathbb{R}$ is of class $C^{2}$, and the first and second derivatives are given by

$$
\begin{gathered}
F^{\prime}(u) v=\int_{\Omega}\left(\varphi_{u}+\nu u\right) v \mathrm{~d} x \\
F^{\prime \prime}(u)\left(v_{1}, v_{2}\right)=\int_{\Omega}\left\{\frac{\partial^{2} L}{\partial y^{2}}\left(x, y_{u}\right) z_{v_{1}} z_{v_{2}}-\frac{\partial^{2} a}{\partial y^{2}}\left(x, y_{u}\right) \varphi_{u} z_{v_{1}} z_{v_{2}}+\nu v_{1} v_{2}\right\} \mathrm{d} x,
\end{gathered}
$$

Copyright (C) by SIAM. Unauthorized reproduction of this article is prohibited. 
where $z_{v_{i}}=G^{\prime}(u) v_{i}, i=1,2$, and $\varphi_{u} \in W^{2, p}(\Omega)$ is the adjoint state defined as the unique solution of

$$
\left\{\begin{aligned}
A^{*} \varphi+\frac{\partial a}{\partial y}\left(x, y_{u}\right) \varphi & =\frac{\partial L}{\partial y}\left(x, y_{u}\right) & & \text { in } \Omega, \\
\varphi & =0 & & \text { on } \Gamma,
\end{aligned}\right.
$$

$A^{*}$ being the adjoint operator of $A$.

Finally, it is obvious that problem $(\mathrm{P})$ has at least one global solution, which belongs to $L^{\infty}(\Omega)$ because of the control constraints. The reader is referred to the book by Tröltzsch [18, Chapter 4.4] for the proof of these results.

Note that under some extra assumptions for $L$ the existence of a solution of (P) in $L^{2}(\Omega)$ can still be proved for $\alpha=-\infty$ or $\beta=+\infty$. For instance, if $L$ is bounded from below, i.e., $L(x, y) \geq C_{L}$, with $C_{L} \in \mathbb{R}$, then the cost functional $J$ is coercive and consequently $(\mathrm{P})$ has again a global solution in $L^{2}(\Omega)$. Indeed, from the first-order optimality conditions we can deduce that this solution is not only in $L^{2}(\Omega)$ but it belongs also to $L^{\infty}(\Omega)$.

Remark 2.4. Theorem 2.2 is also valid for convex polygonal domains $\Omega \subset \mathbb{R}^{2}$. The only difference is that $p$ is not only bounded above by $\bar{p}$, it also depends on the angles of the polygon $\Omega$. Indeed, let $\omega$ be the biggest angle of $\Omega$. Using the results of Grisvard [11, Chapter 4], if $\omega \leq \pi / 2$, then $p$ can be chosen as in Theorem 2.2, only bounded by $\bar{p}$. However, if $\omega>\pi / 2$, then $n / 2<p<\min \left\{\bar{p}, \frac{2}{2-\pi / \omega}\right\}$ is the correct interval. With this modification, Theorem 2.3 also is valid as well as the rest of the results in this paper.

3. First- and second-order optimality conditions. In this section, we will derive the necessary first- and second-order optimality conditions and we will also provide a sufficient second-order condition with a minimal gap with respect to the necessary ones. Since $(\mathrm{P})$ is not a convex problem we will deal with local solutions. As usual, $\bar{u}$ is said to be a local solution of $(\mathrm{P})$ in the $L^{q}(\Omega)$ sense, $1 \leq q \leq+\infty$, if there exists $\varepsilon>0$ such that $\bar{u}$ is a solution of the problem

$$
\min _{u \in \mathbb{K} \cap \bar{B}_{\varepsilon}(\bar{u})} J(u),
$$

where $\bar{B}_{\varepsilon}(\bar{u})$ denotes the closed ball of $L^{q}(\Omega)$ with the center at $\bar{u}$ and the radius $\varepsilon$. The solution is called strict if $\bar{u}$ is the unique global solution of $\left(\mathrm{P}_{\varepsilon}\right)$ for some $\varepsilon>0$. It is immediate to check that if $\bar{u}$ is a local solution in the $L^{q}(\Omega)$ sense for any $1 \leq q<\infty$, then it is also a local solution in the $L^{\infty}(\Omega)$ sense. On the other hand, since $\mathbb{K}$ is bounded in $L^{\infty}(\Omega)$, if $\bar{u}$ is a local solution in the $L^{q}(\Omega)$ sense, for some $1 \leq q<\infty$, then $\bar{u}$ is also a local solution in the $L^{p}(\Omega)$ sense for any $1 \leq p<\infty$. Therefore, we can distinguish two different notions of local minima: $L^{2}(\Omega)$ sense or $L^{\infty}(\Omega)$ sense. The results proved in this paper will hold for either of these two notions of local minima. Therefore, we will not distinguish between these two notions and we will simply speak about local minima.

In the study of the optimality conditions there is a difficulty coming from the nondifferentiability of the function $j(u)=\|u\|_{L^{1}(\Omega)}$ involved in the objective function of (P). Since $j$ is convex and Lipschitz, we can apply some classical results to deduce the first-order conditions. However, the second-order necessary and sufficient optimality conditions, as presented here, are new to the best of our knowledge. The sufficient second-order conditions will be used in the next section to derive the error 
estimates of finite element approximations, which shows their utility. Before stating these optimality conditions we recall some properties of the function $j$. Since $j$ is convex and Lipschitz, the subdifferential in the sense of convex analysis and the generalized gradients introduced by Clarke coincide. Moreover, a simple computation shows that $\lambda \in \partial j(u)$ if and only if

$$
\begin{cases}\lambda(x)=+1 & \text { if } u(x)>0 \\ \lambda(x)=-1 & \text { if } u(x)<0 \\ \lambda(x) \in[-1,+1] & \text { if } u(x)=0\end{cases}
$$

holds a.e. in $\Omega$. Also $j$ has directional derivatives given by

$$
j^{\prime}(u ; v)=\lim _{\rho \searrow 0} \frac{j(u+\rho v)-j(u)}{\rho}=\int_{\Omega_{u}^{+}} v \mathrm{~d} x-\int_{\Omega_{u}^{-}} v \mathrm{~d} x+\int_{\Omega_{u}^{0}}|v| \mathrm{d} x,
$$

for $u, v \in L^{1}(\Omega)$, where $\Omega_{u}^{+}, \Omega_{u}^{-}$and $\Omega_{u}^{0}$ represent the sets of points where $u$ is positive, negative or zero, respectively. Finally, the following relation holds:

$$
\max _{\lambda \in \partial j(u)} \int_{\Omega} \lambda v \mathrm{~d} x=j^{\prime}(u ; v) \leq \frac{j(u+\rho v)-j(u)}{\rho} \quad \forall 0<\rho \leq 1 .
$$

We refer to Clarke [8, Chapter 2] and Bonnans and Shapiro [2, section 2.4.3] for more details. Necessary optimality conditions can be deduced from the abstract results presented in these references and Theorem 2.3.

THEOREM 3.1. If $\bar{u}$ is a local minimum of $(\mathrm{P})$, then there exist $\bar{y}, \bar{\varphi} \in W^{2, \bar{p}}(\Omega)$ and $\bar{\lambda} \in \partial j(\bar{u})$ such that

$$
\begin{aligned}
& \left\{\begin{aligned}
A \bar{y}+a(x, \bar{y}) & =\bar{u} & & \text { in } \Omega, \\
\bar{y} & =0 & & \text { on } \Gamma,
\end{aligned}\right. \\
& \left\{\begin{aligned}
A^{*} \bar{\varphi}+\frac{\partial a}{\partial y}(x, \bar{y}) \bar{\varphi} & =\frac{\partial L}{\partial y}(x, \bar{y}) & & \text { in } \Omega, \\
\bar{\varphi} & =0 & & \text { on } \Gamma,
\end{aligned}\right. \\
& \int_{\Omega}(\bar{\varphi}+\nu \bar{u}+\mu \bar{\lambda})(u-\bar{u}) \mathrm{d} x \geq 0 \quad \forall u \in \mathbb{K} .
\end{aligned}
$$

Corollary 3.2. Let $\bar{u}, \bar{\varphi}$, and $\bar{\lambda}$ be as in the previous theorem; then the following relations hold:

$$
\begin{aligned}
& \bar{u}(x)=\operatorname{Proj}_{[\alpha, \beta]}\left(-\frac{1}{\nu}(\bar{\varphi}(x)+\mu \bar{\lambda}(x))\right), \\
& \bar{u}(x)=0 \Leftrightarrow|\bar{\varphi}(x)| \leq \mu, \\
& \bar{\lambda}(x)=\operatorname{Proj}_{[-1,+1]}\left(-\frac{1}{\mu} \bar{\varphi}(x)\right) .
\end{aligned}
$$

Moreover, from the first and last representation formulas it follows that $\bar{u}, \bar{\lambda} \in C^{0,1}(\bar{\Omega})$ and $\bar{\lambda}$ is unique for any fixed local minimum $\bar{u}$. 
Proof. The derivation of the formula (3.5a) is standard in control theory. Now, from (3.1), (3.5a), and the fact that $\alpha<0<\beta$, we get

$$
\begin{aligned}
& \bar{u}(x)=0 \Rightarrow \bar{\varphi}(x)+\mu \bar{\lambda}(x)=0 \Rightarrow|\bar{\varphi}(x)| \leq \mu, \\
& \bar{u}(x)>0 \Rightarrow \bar{\lambda}(x)=+1 \text { and } \bar{\varphi}(x)+\mu \bar{\lambda}(x)<0 \Rightarrow \bar{\varphi}(x)<-\mu,
\end{aligned}
$$

and analogously we deduce that if $\bar{u}(x)<0$, then $\bar{\varphi}(x)>\mu$. These three properties are equivalent to (3.5b). Let us prove (3.5c). Taking into account (3.1), (3.5b), and (3.5a), we obtain

$$
\begin{aligned}
& \bar{\varphi}(x)>\mu \Rightarrow \bar{u}(x)<0 \Rightarrow \bar{\lambda}(x)=-1 \Rightarrow \bar{\lambda}(x)=\operatorname{Proj}_{[-1,+1]}\left(-\frac{1}{\mu} \bar{\varphi}(x)\right), \\
& |\bar{\varphi}(x)| \leq \mu \Rightarrow \bar{u}(x)=0 \Rightarrow \bar{\varphi}(x)+\mu \bar{\lambda}(x)=0 \Rightarrow \bar{\lambda}(x)=\operatorname{Proj}_{[-1,+1]}\left(-\frac{1}{\mu} \bar{\varphi}(x)\right) .
\end{aligned}
$$

For the case $\bar{\varphi}(x)<-\mu$ we can proceed as for the case $\bar{\varphi}(x)>\mu$, which completes the proof of $(3.5 \mathrm{c})$.

The Lipschitz property of $\bar{\lambda}$ follows from (3.5c) and from the fact that $\bar{\varphi} \in$ $W^{2, \bar{p}}(\Omega) \hookrightarrow C^{1}(\bar{\Omega})$. Finally, (3.5a) leads to the Lipschitz regularity of $\bar{u}$.

Remark 3.3. Let us point out that the relation (3.5b) implies the sparsity of local optimal controls. This property was observed by [17] and it continues to hold in the cases $\alpha=0$ or $\beta=0$. Indeed, if $\alpha=0$, it is easy to deduce from (3.5a) that $\bar{u}(x)=0$ if and only if $\bar{\varphi}(x) \geq-\mu$, which also implies the sparsity. For $\beta=0$, we have that $\bar{u}(x)=0$ if and only if $\bar{\varphi}(x) \leq+\mu$.

In order to address the second-order optimality conditions we need to introduce the critical cone. Given a control $\bar{u} \in \mathbb{K}$ for which there exists $\bar{\lambda} \in \partial j(\bar{u})$ satisfying (3.4), we define

$$
C_{\bar{u}}=\left\{v \in L^{2}(\Omega) \text { satisfying }(3.7) \text { and } F^{\prime}(\bar{u}) v+\mu j^{\prime}(\bar{u} ; v)=0\right\}
$$

with

$$
\begin{cases}v(x) \geq 0 & \text { if } \bar{u}(x)=\alpha \\ v(x) \leq 0 & \text { if } \bar{u}(x)=\beta\end{cases}
$$

Proposition 3.4. The set $C_{\bar{u}}$ is a closed, convex cone in $L^{2}(\Omega)$.

Before proving this proposition we have to establish the following lemma.

Lemma 3.5. Let $\bar{u} \in \mathbb{K}$ satisfy (3.4) along with some $\bar{\lambda} \in \partial j(\bar{u})$. Let $v \in L^{2}(\Omega)$ fulfill (3.7). Then

$$
F^{\prime}(\bar{u}) v+\mu j^{\prime}(\bar{u} ; v) \geq F^{\prime}(\bar{u}) v+\mu \int_{\Omega} \bar{\lambda}(x) v(x) \mathrm{d} x \geq 0 .
$$

Moreover, if $v \in C_{\bar{u}}$, then

$$
F^{\prime}(\bar{u}) v+\mu \int_{\Omega} \bar{\lambda}(x) v(x) \mathrm{d} x=0 \quad \text { and } \quad j^{\prime}(\bar{u} ; v)=\int_{\Omega} \bar{\lambda}(x) v(x) \mathrm{d} x .
$$

Proof. The first inequality of (3.8) is an immediate consequence of (3.3). Let us prove the second inequality. For every $k \in \mathbb{N}$ we define

$$
v_{k}(x)=\left\{\begin{array}{cl}
0 & \text { if } \alpha<\bar{u}(x)<\alpha+\frac{1}{k} \text { or } \beta-\frac{1}{k}<\bar{u}(x)<\beta, \\
\operatorname{Proj}_{[-k,+k]}(v(x)) & \text { otherwise, }
\end{array}\right.
$$

Copyright $@$ by SIAM. Unauthorized reproduction of this article is prohibited. 
and $\rho_{k}=1 / k^{2}$. Then it is easy to check that $\bar{u}+\rho v_{k} \in \mathbb{K}$ for every $0<\rho<\rho_{k}$. On the other hand, $\left|v_{k}(x)\right| \leq|v(x)|$ and $v_{k}(x) \rightarrow v(x)$ hold for almost all $x \in \Omega$; therefore $v_{k} \rightarrow v$ in $L^{2}(\Omega)$. Now, from (3.4c) we infer

$$
\rho\left(F^{\prime}(\bar{u}) v_{k}+\mu \int_{\Omega} \bar{\lambda}(x) v_{k}(x) \mathrm{d} x\right)=\int_{\Omega}(\bar{\varphi}+\nu \bar{u}+\mu \bar{\lambda})\left(\left[\bar{u}+\rho v_{k}\right]-\bar{u}\right) \geq 0 .
$$

Finally, dividing the previous expression by $\rho$ and passing to the limit as $k \rightarrow \infty$, we obtain the second inequality of (3.8).

Identities (3.9) are an obvious consequence of (3.8) and the equality satisfied by the elements of $C_{\bar{u}}$.

Remark 3.6. Let us observe that for any $v \in L^{2}(\Omega)$ satisfying

$$
j^{\prime}(\bar{u} ; v)=\int_{\Omega} \bar{\lambda}(x) v(x) \mathrm{d} x,
$$

the relations (3.1) and (3.2) imply that

$$
\int_{\Omega_{\bar{u}}^{0}}|v(x)| \mathrm{d} x=\int_{\Omega_{\bar{u}}^{0}} \bar{\lambda}(x) v(x) \mathrm{d} x \Rightarrow \int_{\Omega_{\bar{u}}^{0}}(|v(x)|-\bar{\lambda}(x) v(x)) \mathrm{d} x=0,
$$

which leads to $|v(x)|=\bar{\lambda}(x) v(x)$ for almost all $x \in \Omega_{\bar{u}}^{0}$. In particular, (3.9) implies that this identity holds for all the elements of $C_{\bar{u}}$.

Proof of Proposition 3.4. It is obvious that $C_{\bar{u}}$ is a closed cone of $L^{2}(\Omega)$. Let us prove that it is convex. Given $v_{1}, v_{2} \in C_{\bar{u}}$ and $0<t<1$, it is clear that $v=$ $t v_{1}+(1-t) v_{2}$ satisfies (3.7) and using the convexity of $j$ we get

$$
F^{\prime}(\bar{u}) v+\mu j(\bar{u} ; v) \leq t\left[F^{\prime}(\bar{u}) v_{1}+\mu j^{\prime}\left(\bar{u} ; v_{1}\right)\right]+(1-t)\left[F^{\prime}(\bar{u}) v_{2}+\mu j^{\prime}\left(\bar{u} ; v_{2}\right)\right]=0
$$

The contrary inequality is a consequence of Lemma 3.5; hence $v \in C_{\bar{u}}$.

Let us introduce some notation. We define

$$
\bar{d}(x)=\bar{\varphi}(x)+\nu \bar{u}(x)+\mu \bar{\lambda}(x) \in C^{0,1}(\bar{\Omega}),
$$

the Lipschitz regularity of $\bar{d}$ being a consequence of the regularity properties established in Corollary 3.2. From (3.5a) we deduce as usual

$$
\left\{\begin{array} { c c } 
{ \overline { u } ( x ) = \alpha } & { \Rightarrow \overline { d } ( x ) \geq 0 , } \\
{ \overline { u } ( x ) = \beta } & { \Rightarrow \overline { d } ( x ) \leq 0 , } \\
{ \alpha < \overline { u } ( x ) < \beta } & { \Rightarrow \overline { d } ( x ) = 0 }
\end{array} \quad \text { and } \quad \left\{\begin{array}{cll}
\bar{d}(x)>0 & \Rightarrow \bar{u}(x)=\alpha, \\
\bar{d}(x)<0 & \Rightarrow & \bar{u}(x)=\beta
\end{array}\right.\right.
$$

a.e. in $\Omega$.

On the other hand, from (3.9) we have

$$
\int_{\Omega} \bar{d}(x) v(x) \mathrm{d} x=F^{\prime}(\bar{u}) v+\mu \int_{\Omega} \bar{\lambda}(x) v(x) \mathrm{d} x=0 \quad \forall v \in C_{\bar{u}} .
$$

This identity, along with (3.7) and (3.11), leads to

$$
\int_{\Omega}|\bar{d}(x) v(x)| \mathrm{d} x=\int_{\Omega} \bar{d}(x) v(x) \mathrm{d} x=0 \quad \Rightarrow \quad \bar{d}(x) v(x) \equiv 0 \quad \forall v \in C_{\bar{u}} .
$$

Now, we can formulate the second-order necessary optimality conditions as follows.

Copyright $\odot$ by SIAM. Unauthorized reproduction of this article is prohibited. 
THEOREM 3.7. Let us assume that $\bar{u}$ is a local minimum of $(\mathrm{P})$; then $F^{\prime \prime}(\bar{u}) v^{2} \geq 0$ for every $v \in C_{\bar{u}}$.

Proof. Given $v \in C_{\bar{u}}$ we define for every $k \in \mathbb{N}$

$$
v_{k}(x)=\left\{\begin{array}{l}
0 \text { if } \alpha<\bar{u}(x)<\alpha+\frac{1}{k} \text { or } \beta-\frac{1}{k}<\bar{u}(x)<\beta \text { or } 0<|\bar{u}(x)|<\frac{1}{k}, \\
\operatorname{Proj}_{[-k,+k]}(v(x)) \text { otherwise, }
\end{array}\right.
$$

and $\rho_{k}=1 / k^{2}$. Then, as in the proof of Lemma 3.5, we have that $\bar{u}+\rho v_{k} \in \mathbb{K}$ for every $0<\rho<\rho_{k}$ and $v_{k} \rightarrow v$ in $L^{2}(\Omega)$. On the other hand, it is obvious that $v_{k}(x)=0$ whenever $v(x)=0$, and the sign of $v_{k}(x)$ coincides with the sign of $v(x)$ whenever $v_{k}(x) \neq 0$.

Let $\bar{\lambda}$ be the unique element of $\partial j(\bar{u})$ associated with $\bar{u}$; see Corollary 3.2. Then, by (3.9), $j^{\prime}(\bar{u} ; v)=\int_{\Omega} \bar{\lambda}(x) v(x) \mathrm{d} x$ holds. Consequently, we have $|v(x)|=\bar{\lambda}(x) v(x)$ for $x \in \Omega_{\bar{u}}^{0}$, as observed in Remark 3.6. Moreover, $\bar{d}(x) v(x) \equiv 0$ holds by (3.12). Owing to the sign condition for $v_{k}$, we obtain

$$
\left|v_{k}(x)\right|=\bar{\lambda}(x) v_{k}(x) \text { for a.a. } x \in \Omega_{\bar{u}}^{0} \text { and } \bar{d}(x) v_{k}(x) \equiv 0 \text { for a.a. } x \in \Omega .
$$

Let us analyze the case where $x \in \Omega_{\bar{u}}^{+}$. In this situation, since $\rho\left|v_{k}(x)\right|<\rho_{k} k \leq 1 / k$ and $v_{k}(x)=0$ if $0<\bar{u}(x)<1 / k$, we necessarily have that $\bar{u}(x)+\rho v_{k}(x) \geq 0$ which, along with the fact that $\bar{\lambda}(x)=1$, leads to

$$
\left|\bar{u}(x)+\rho v_{k}(x)\right|-|\bar{u}(x)|=\rho v_{k}(x)=\rho \bar{\lambda}(x) v_{k}(x) .
$$

Analogously, we obtain that $\left|\bar{u}(x)+\rho v_{k}(x)\right|-|\bar{u}(x)|=-\rho v_{k}(x)=\rho \bar{\lambda}(x) v_{k}(x)$ when $x \in \Omega_{\bar{u}}^{-}$. Thus, from this identity, (3.13), and (3.14) we conclude that

$$
j\left(\bar{u}+\rho v_{k}\right)-j(\bar{u})=\rho \int_{\Omega} \bar{\lambda}(x) v_{k}(x) \mathrm{d} x \quad \forall 0<\rho<\rho_{k} .
$$

On the other hand, the second identity of (3.13) can be written as

$$
F^{\prime}(\bar{u}) v_{k}+\mu \int_{\Omega} \bar{\lambda}(x) v_{k}(x) \mathrm{d} x=\int_{\Omega} \bar{d}(x) v_{k}(x) \mathrm{d} x=0 .
$$

Now, using the fact that $\bar{u}$ is a local minimum and taking into account (3.15) and (3.16), we infer for all $\rho>0$ sufficiently small that

$$
\begin{aligned}
0 & \leq J\left(\bar{u}+\rho v_{k}\right)-J(\bar{u}) \\
& =\left[F(\bar{u})+\rho F^{\prime}(\bar{u}) v_{k}+\frac{\rho^{2}}{2} F^{\prime \prime}(\bar{u}) v_{k}^{2}+o\left(\rho^{2}\right)+\mu j\left(\bar{u}+\rho v_{k}\right)\right]-[F(\bar{u})+\mu j(\bar{u})] \\
& =\rho F^{\prime}(\bar{u}) v_{k}+\frac{\rho^{2}}{2} F^{\prime \prime}(\bar{u}) v_{k}^{2}+o\left(\rho^{2}\right)+\mu\left[j\left(\bar{u}+\rho v_{k}\right)-j(\bar{u})\right] \\
& =\frac{\rho^{2}}{2} F^{\prime \prime}(\bar{u}) v_{k}^{2}+o\left(\rho^{2}\right)+\rho\left\{F^{\prime}(\bar{u}) v_{k}+\mu \int_{\Omega} \bar{\lambda}(x) v_{k}(x) \mathrm{d} x\right\} \\
& =\frac{\rho^{2}}{2} F^{\prime \prime}(\bar{u}) v_{k}^{2}+o\left(\rho^{2}\right) .
\end{aligned}
$$

Dividing the last expression by $\rho^{2} / 2$ and letting $\rho \searrow 0$, we obtain $F^{\prime \prime}(\bar{u}) v_{k}^{2} \geq 0$. Finally, passing to the limit when $k \rightarrow \infty$, we conclude that $F^{\prime \prime}(\bar{u}) v^{2} \geq 0$. 
We finish the section by proving the sufficient condition in Theorem 3.9 with a minimal gap w.r.t. the necessary one proved in Theorem 3.7. Before we do so, we recall that a natural assumption would be the positivity of the second derivative $F^{\prime \prime}(\bar{u})$ on the critical cone $C_{\bar{u}}$. Due to the $L^{2}$ regularization term, this already implies that $F^{\prime \prime}(\bar{u})$ is uniformly positive even on a larger cone. This is established in the next theorem. Moreover, this second equivalent condition will be used for the numerical analysis in section 4 .

TheOREm 3.8. Let $\bar{u} \in \mathbb{K}$ and $\bar{\lambda} \in \partial j(\bar{u})$ such that (3.4) hold. Then the following statements are equivalent:

1. $F^{\prime \prime}(\bar{u}) v^{2}>0$ for all $v \in C_{\bar{u}} \backslash\{0\}$.

2. There exist $\tau>0$ and $\delta>0$ such that $F^{\prime \prime}(\bar{u}) v^{2} \geq \delta\|v\|_{L^{2}(\Omega)}^{2}$ for all $v \in C_{\bar{u}}^{\tau}$, where

$$
C_{\bar{u}}^{\tau}=\left\{v \in L^{2}(\Omega) \text { satisfying (3.7) and } F^{\prime}(\bar{u}) v+\mu j^{\prime}(\bar{u} ; v) \leq \tau\|v\|_{L^{2}(\Omega)}\right\} .
$$

Proof. Since $C_{\bar{u}} \subset C_{\bar{u}}^{\tau}$, it is obvious that the second condition implies the first one. Let us prove the other implication. We will proceed by contradiction. Then, we assume that the first condition holds, but not the second. Hence, there exists a sequence $\left\{v_{k}\right\}_{k=1}^{\infty}$ such that

$$
v_{k} \in C_{\bar{u}}^{1 / k} \quad \text { and } \quad F^{\prime \prime}(\bar{u}) v_{k}^{2}<\frac{1}{k}\left\|v_{k}\right\|_{L^{2}(\Omega)}^{2} .
$$

Since $C_{\bar{u}}^{1 / k}$ is a cone, we can divide $v_{k}$ by its $L^{2}(\Omega)$ norm and, by taking a subsequence if necessary, we can assume that

$$
v_{k} \in C_{\bar{u}}^{1 / k}, \quad F^{\prime \prime}(\bar{u}) v_{k}^{2}<\frac{1}{k}, \quad \text { and } \quad v_{k} \rightarrow v \text { in } L^{2}(\Omega) .
$$

Since $v_{k} \in C_{\bar{u}}^{1 / k}$, then $v_{k}$ satisfies the sign conditions (3.7), and therefore $v$ also does. Then, (3.8) implies

$$
F^{\prime}(\bar{u}) v+\mu j^{\prime}(\bar{u} ; v) \geq 0 .
$$

On the other hand, using again that $v_{k} \in C_{\bar{u}}^{1 / k}$, we get

$$
F^{\prime}(\bar{u}) v+\mu j^{\prime}(\bar{u} ; v) \leq \liminf _{k \rightarrow \infty}\left\{F^{\prime}(\bar{u}) v_{k}+\mu j^{\prime}\left(\bar{u} ; v_{k}\right)\right\} \leq \liminf _{k \rightarrow \infty} \frac{1}{k}=0 .
$$

Inequalities (3.18) and (3.19), along with the sign condition (3.7) satisfied by $v$, imply that $v \in C_{\bar{u}}$. Now, we observe that Theorem 2.2 and the compactness of the embedding $W^{2, p}(\Omega) \subset C(\bar{\Omega})$ imply the compactness of the linear operator $v \in L^{2}(\Omega) \rightarrow$ $z_{v}=G^{\prime}(\bar{u}) v \in C(\bar{\Omega})$. From this property along with the continuity and convexity of $v \in L^{2}(\Omega) \rightarrow\|v\|_{L^{2}(\Omega)}^{2}$, and the expression $(2.8)$, we conclude that $F^{\prime \prime}(\bar{u}): L^{2}(\Omega) \rightarrow \mathbb{R}$ is a weakly lower semicontinuous quadratic functional. Then, from (3.17) we infer

$$
F^{\prime \prime}(\bar{u}) v^{2} \leq \liminf _{k \rightarrow \infty} F^{\prime \prime}(\bar{u}) v_{k}^{2} \leq \limsup _{k \rightarrow \infty} F^{\prime \prime}(\bar{u}) v_{k}^{2} \leq 0,
$$

which is only possible if $v=0$ because of condition 1 of Theorem 3.8; therefore, $F^{\prime \prime}(\bar{u}) v_{k}^{2} \rightarrow 0$. However, $v_{k} \rightarrow 0$ in $L^{2}(\Omega)$ implies the strong convergence $z_{v_{k}} \rightarrow 0$ in $W^{1, \bar{p}}(\Omega)$. Therefore,

$$
\begin{aligned}
F^{\prime \prime}(\bar{u}) v_{k}^{2} & =\int_{\Omega}\left\{\frac{\partial^{2} L}{\partial y^{2}}(x, \bar{y}) z_{v_{k}}^{2}-\frac{\partial^{2} a}{\partial y^{2}}(x, \bar{y}) \bar{\varphi} z_{v_{k}}^{2}+\nu v_{k}^{2}\right\} \mathrm{d} x \\
& =\int_{\Omega}\left\{\frac{\partial^{2} L}{\partial y^{2}}(x, \bar{y}) z_{v_{k}}^{2}-\frac{\partial^{2} a}{\partial y^{2}}(x, \bar{y}) \bar{\varphi} z_{v_{k}}^{2}\right\} \mathrm{d} x+\nu
\end{aligned}
$$

Copyright $\odot$ by SIAM. Unauthorized reproduction of this article is prohibited. 
converges to $\nu$, which is a contradiction.

Finally, we prove the sufficient second-order optimality condition.

ThEOREM 3.9. Let $\bar{u} \in \mathbb{K}$ and $\bar{\lambda} \in \partial j(\bar{u})$ such that (3.4) hold. Furthermore, let us assume that $F^{\prime \prime}(\bar{u}) v^{2}>0$ for all $v \in C_{\bar{u}} \backslash\{0\}$; then there exist $\delta>0$ and $\varepsilon>0$ such that

$$
J(\bar{u})+\frac{\delta}{4}\|u-\bar{u}\|_{L^{2}(\Omega)}^{2} \leq J(u) \quad \forall u \in \mathbb{K} \cap B_{\varepsilon}(\bar{u}),
$$

where $B_{\varepsilon}(\bar{u})$ denotes the $L^{2}(\Omega)$ ball of center $\bar{u}$ and radius $\varepsilon$.

Proof. Let $\varepsilon>0$ and $u \in \mathbb{K} \cap B_{\varepsilon}(\bar{u})$ be given. We define

$$
\rho=\|u-\bar{u}\|_{L^{2}(\Omega)} \leq \varepsilon, \quad v=\frac{1}{\rho}(u-\bar{u}) .
$$

A second-order Taylor expansion of $F$ yields

$F(u)=F(\bar{u})+\rho F^{\prime}(\bar{u}) v+\frac{\rho^{2}}{2} F^{\prime \prime}(\bar{u}) v^{2}+\rho^{2} \int_{0}^{1}(1-t)\left(F^{\prime \prime}(\bar{u}+t \rho v)-F^{\prime \prime}(\bar{u})\right) v^{2} \mathrm{~d} t$.

It follows from the continuity of $F^{\prime \prime}$ that the last term is of order $o\left(\rho^{2}\right)$. Using the convexity of $j$, this shows

$$
J(u)-J(\bar{u}) \geq \rho\left(F^{\prime}(\bar{u}) v+\mu j^{\prime}(\bar{u}, v)\right)+\frac{\rho^{2}}{2} F^{\prime \prime}(\bar{u}) v^{2}+o\left(\rho^{2}\right) .
$$

In case $v \in C_{\bar{u}}^{\tau}$, Theorem 3.8 and Lemma 3.5 imply

$$
J(u)-J(\bar{u}) \geq \frac{\rho^{2}}{2} \delta+o\left(\rho^{2}\right),
$$

where $\delta>0$.

On the other hand, if $v \notin C_{\bar{u}}^{\tau}$, we have $F^{\prime}(\bar{u}) v+\mu j^{\prime}(\bar{u}, v) \geq \tau$, since $v$ satisfies (3.7). Hence, we have

$$
J(u)-J(\bar{u}) \geq \rho \tau-\frac{\rho^{2}}{2}\left\|F^{\prime \prime}(\bar{u})\right\|+o\left(\rho^{2}\right) .
$$

This shows (3.20) for $\varepsilon$ sufficiently small.

4. Finite element approximation of $(\mathbf{P})$. The goal of this section is to study the approximation of problem $(\mathrm{P})$ by finite elements. Both the state and the controls will be discretized. We prove the convergence of the discretization and derive some associated error estimates. To this aim, we consider a family of triangulations $\left\{\mathcal{T}_{h}\right\}_{h>0}$ of $\bar{\Omega}$, defined in the standard way, e.g., in [3, Chapter 3.3]. Due to the assumption that $\Omega$ has a smooth boundary, the triangulation covers a polygonal approximation $\Omega_{h}$. With each element $T \in \mathcal{T}_{h}$, we associate two parameters $\rho(T)$ and $\sigma(T)$, where $\rho(T)$ denotes the diameter of the set $T$ and $\sigma(T)$ is the diameter of the largest ball contained in $T$. Define the size of the mesh by $h=\max _{T \in \mathcal{T}_{h}} \rho(T)$. To simplify the presentation of the results, in what follows we suppose that $\Omega$ is convex. We also assume that the following regularity assumptions on the triangulation are satisfied which are standard in the context of $L^{\infty}$ error estimates.

(i) There exist two positive constants $\rho$ and $\sigma$ such that

$$
\frac{\rho(T)}{\sigma(T)} \leq \sigma, \quad \frac{h}{\rho(T)} \leq \rho
$$

hold for all $T \in \mathcal{T}_{h}$ and all $h>0$. 
(ii) Define $\bar{\Omega}_{h}=\cup_{T \in \mathcal{T}_{h}} T$, and let $\Omega_{h}$ and $\Gamma_{h}$ denote its interior and its boundary, respectively. We assume that $\bar{\Omega}_{h}$ is convex and that the vertices of $\mathcal{T}_{h}$ placed on the boundary $\Gamma_{h}$ are points of $\Gamma$. From [15, estimate (5.2.19)] we know that

$$
\left|\Omega \backslash \Omega_{h}\right| \leq C h^{2} .
$$

We will use piecewise linear approximations for the states; thus we set

$$
Y_{h}=\left\{y_{h} \in C(\bar{\Omega}) \mid y_{h \mid T} \in \mathcal{P}_{1} \forall T \in \mathcal{T}_{h} \text {, and } y_{h}=0 \text { on } \bar{\Omega} \backslash \Omega_{h}\right\},
$$

where $\mathcal{P}_{1}$ is the space of polynomials of degree less than or equal to 1.

The discrete version of (2.1) is defined as follows:

$$
\left\{\begin{array}{l}
\text { Find } y_{h} \in Y_{h} \text { such that } \forall z_{h} \in Y_{h}, \\
\int_{\Omega_{h}}\left[\sum_{i, j=1}^{n} a_{i j} \partial_{x_{i}} y_{h} \partial_{x_{j}} z_{h}+a\left(x, y_{h}\right) z_{h}\right] \mathrm{d} x=\int_{\Omega_{h}} u z_{h} \mathrm{~d} x .
\end{array}\right.
$$

Thanks to the monotonicity of the nonlinear term of (4.1) and using Brouwer's fixed point theorem, it is easy to prove the existence and uniqueness of a solution $y_{h}(u)$ of (4.1) for any $u \in L^{2}\left(\Omega_{h}\right)$.

Now, we define the space of discrete controls by

$$
\mathcal{U}_{h}=\left\{u_{h} \in L^{2}\left(\Omega_{h}\right):\left.u_{h}\right|_{T}=\text { constant } \forall T \in \mathcal{T}_{h}\right\} .
$$

Every element $u_{h} \in \mathcal{U}_{h}$ can be written in the form

$$
u_{h}=\sum_{T \in \mathcal{T}_{h}} u_{T} \chi_{T},
$$

where $\chi_{T}$ is the characteristic function of $T$. The set of discrete feasible controls is given by

$$
\mathbb{K}_{h}=\left\{u_{h} \in \mathcal{U}_{h}: \alpha \leq u_{T} \leq \beta \quad \forall T \in \mathcal{T}_{h}\right\}
$$

Finally, the discrete control problem is formulated as follows:

$$
\left\{\begin{array}{l}
\min J_{h}\left(u_{h}\right)=F_{h}\left(u_{h}\right)+\mu j_{h}\left(u_{h}\right), \\
u_{h} \in \mathbb{K}_{h},
\end{array}\right.
$$

where $F_{h}: L^{2}\left(\Omega_{h}\right) \longrightarrow \mathbb{R}$ and $j_{h}: \mathcal{U}_{h} \longrightarrow \mathbb{R}$ are defined by

$$
F_{h}(u)=\int_{\Omega_{h}} L\left(x, y_{h}\left(u_{h}\right)\right) \mathrm{d} x+\frac{\nu}{2} \int_{\Omega_{h}} u_{h}^{2} \mathrm{~d} x \text { and } j_{h}\left(u_{h}\right)=\int_{\Omega_{h}}\left|u_{h}\right| \mathrm{d} x .
$$

It is immediate that $\left(\mathrm{P}_{h}\right)$ has at least one solution and we have the following first-order optimality conditions analogous to those of problem (P); see Theorem 3.1.

THEOREM 4.1. If $\bar{u}_{h}$ is a local minimum of problem $\left(\mathrm{P}_{h}\right)$, then there exist $\bar{y}_{h}, \bar{\varphi}_{h} \in$ 
$Y_{h}$ and $\bar{\lambda}_{h} \in \partial j_{h}\left(u_{h}\right)$ such that

(4.2a)

$$
\int_{\Omega_{h}}\left[\sum_{i, j=1}^{n} a_{i j} \partial_{x_{i}} \bar{y}_{h} \partial_{x_{j}} z_{h}+a\left(x, \bar{y}_{h}\right) z_{h}\right] \mathrm{d} x=\int_{\Omega_{h}} \bar{u}_{h} z_{h} \mathrm{~d} x \quad \forall z_{h} \in Y_{h},
$$

$$
\int_{\Omega_{h}}\left[\sum_{i, j=1}^{n} a_{i j} \partial_{x_{i}} z_{h} \partial_{x_{j}} \bar{\varphi}_{h}+\frac{\partial a}{\partial y}\left(x, \bar{y}_{h}\right) \bar{\varphi}_{h} z_{h}\right] \mathrm{d} x=\int_{\Omega_{h}} \frac{\partial L}{\partial y}\left(x, \bar{y}_{h}\right) z_{h} \mathrm{~d} x \quad \forall z_{h} \in Y_{h},
$$

$$
\int_{\Omega_{h}}\left(\bar{\varphi}_{h}+\nu \bar{u}_{h}+\mu \bar{\lambda}_{h}\right)\left(u_{h}-\bar{u}_{h}\right) \mathrm{d} x \geq 0 \quad \forall u_{h} \in \mathbb{K}_{h} .
$$

It is an easy exercise to check that $\bar{\lambda}_{h}$ can be written in the form

$$
\bar{\lambda}_{h}=\sum_{T \in \mathcal{T}_{h}} \bar{\lambda}_{T} \chi_{T} \quad \text { with }\left\{\begin{array}{cc}
\bar{\lambda}_{T}=+1 & \text { if } \bar{u}_{T}>0 \\
\bar{\lambda}_{T}=-1 & \text { if } \bar{u}_{T}<0 \\
\bar{\lambda}_{T} \in[-1,+1] & \text { if } \bar{u}_{T}=0
\end{array}\right.
$$

where $\bar{u}_{T}$ are the coefficients of $\bar{u}_{h}$,

$$
\bar{u}_{h}=\sum_{T \in \mathcal{T}_{h}} \bar{u}_{T} \chi_{T} .
$$

Inequality $(4.2 \mathrm{c})$ can be written in the form

$$
\sum_{T \in \mathcal{T}_{h}}\left(\int_{T} \bar{\varphi}_{h} \mathrm{~d} x+|T|\left[\nu \bar{u}_{T}+\mu \bar{\lambda}_{T}\right]\right)\left(u_{T}-\bar{u}_{T}\right) \geq 0 \quad \forall \alpha \leq u_{T} \leq \beta,
$$

which leads to the representation formula

$$
\bar{u}_{T}=\operatorname{Proj}_{[\alpha, \beta]}\left(-\frac{1}{\nu}\left[\frac{1}{|T|} \int_{T} \bar{\varphi}_{h} \mathrm{~d} x+\mu \bar{\lambda}_{T}\right]\right) .
$$

Using (4.3) and (4.4a) and arguing as in the proof of Corollary 3.2, we can prove

$$
\bar{u}_{T}=0 \Leftrightarrow \frac{1}{|T|}\left|\int_{T} \bar{\varphi}_{h} \mathrm{~d} x\right| \leq \mu \quad \forall T \in \mathcal{T}_{h}
$$

and

$$
\bar{\lambda}_{T}=\operatorname{Proj}_{[-1,+1]}\left(-\frac{1}{\mu|T|} \int_{T} \bar{\varphi}_{h} \mathrm{~d} x\right) \quad \forall T \in \mathcal{T}_{h} .
$$

As for the infinite dimensional case, this representation formula implies that $\bar{\lambda}_{h}$ is unique for a given local minimum $\bar{u}_{h}$.

On the other hand, defining

$$
\bar{d}_{h}(x)=\bar{\varphi}_{h}(x)+\nu \bar{u}_{h}(x)+\mu \bar{\lambda}_{h}(x),
$$

Copyright $@$ by SIAM. Unauthorized reproduction of this article is prohibited. 
we get the analogous relations to (3.11)

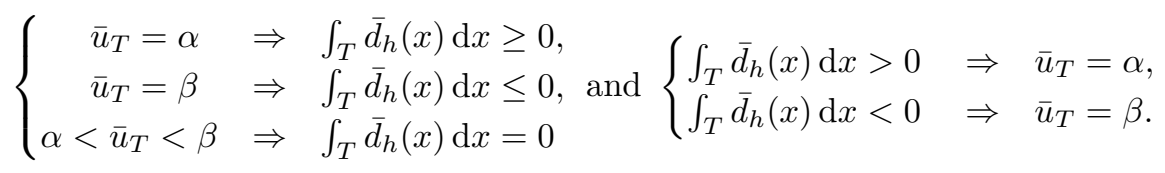

The rest of the section is divided into two parts. In the first part, we prove that the family of problems $\left(\mathrm{P}_{h}\right)$ realizes a convergent approximation of problem $(\mathrm{P})$ in a two-fold sense: global solutions of $\left(\mathrm{P}_{h}\right)$ converge to global solutions of $(\mathrm{P})$ and strict local solutions of $(\mathrm{P})$ can be approximated by local solutions of $\left(\mathrm{P}_{h}\right)$. In the second part of the section, we prove some error estimates for these approximations.

4.1. Convergence of the discretizations. Before proving the convergence of the solutions of $\left(\mathrm{P}_{h}\right)$ to solutions of $(\mathrm{P})$, we need to establish some convergence properties of the finite element approximation of the state and adjoint state equations. The next result is well known; see [1], [5], and [16] and the references therein.

Lemma 4.2. Let $u, v \in L^{\infty}(\Omega)$ fulfill $\|u\|_{L^{\infty}(\Omega)}+\|v\|_{L^{\infty}(\Omega)} \leq M$, and let $y_{u}$, $y_{h}(v), \varphi_{u}$, and $\varphi_{h}(v)$ be the solutions of (2.1), (4.1) (with u replaced by $v$ ), (2.9), and $(4.2 \mathrm{~b})\left(\right.$ with $\bar{y}_{h}$ replaced by $\left.y_{h}(v)\right)$, respectively. Then the following a priori estimates hold:

$$
\left\|y_{u}-y_{h}(v)\right\|_{H^{1}\left(\Omega_{h}\right)}+\left\|\varphi_{u}-\varphi_{h}(v)\right\|_{H^{1}\left(\Omega_{h}\right)} \leq C\left(h+\|u-v\|_{L^{2}(\Omega)}\right),
$$

$$
\left\|y_{u}-y_{h}(v)\right\|_{L^{2}\left(\Omega_{h}\right)}+\left\|\varphi_{u}-\varphi_{h}(v)\right\|_{L^{2}\left(\Omega_{h}\right)} \leq C\left(h^{2}+\|u-v\|_{L^{2}(\Omega)}\right),
$$

$$
\left\|y_{u}-y_{h}(v)\right\|_{L^{\infty}\left(\Omega_{h}\right)}+\left\|\varphi_{u}-\varphi_{h}(v)\right\|_{L^{\infty}\left(\Omega_{h}\right)} \leq C\left(h^{2-n / \bar{p}}|\log h|+\|u-v\|_{L^{2}(\Omega)}\right) .
$$

Moreover, if $u_{h} \rightarrow u$ weakly in $L^{p}(\Omega)$, with $p>n / 2$, then $y_{h}\left(u_{h}\right) \rightarrow y_{u}$ and $\varphi_{h}\left(u_{h}\right) \rightarrow$ $\varphi_{u}$ in $H_{0}^{1}(\Omega) \cap C(\bar{\Omega})$ strongly and $J(u) \leq \liminf _{h \rightarrow 0} J_{h}\left(u_{h}\right)$ holds.

Remark 4.3. Given a sequence $\left\{u_{h}\right\}_{h>0}$, with $u_{h} \in L^{q}\left(\Omega_{h}\right)$ and $1 \leq q \leq+\infty$, we say that $u_{h} \rightarrow u$ weakly in $L^{q}(\Omega)$ (respectively, weakly* in $L^{\infty}(\Omega)$ if $q=\infty$ ) if $u \in L^{q}(\Omega)$ and

$$
\int_{\Omega_{h}} g u_{h} \mathrm{~d} x \rightarrow \int_{\Omega} g u \mathrm{~d} x \quad \forall g \in L^{q^{\prime}}(\Omega) .
$$

The above definition is equivalent to the weak (or weak ${ }^{*}$ ) convergence of any extension $\left\{\tilde{u}_{h}\right\}_{h>0} \subset L^{q}(\Omega)$ of $\left\{u_{h}\right\}_{h>0}$ such that $\left.\tilde{u}_{h}\right|_{\Omega \backslash \Omega_{h}} \stackrel{(*)}{=} 0$ when $h \rightarrow 0$. Since $\left|\Omega \backslash \Omega_{h}\right| \rightarrow 0$ when $h \rightarrow 0$, this is, in particular, the case if we extend $u_{h}$ by an $L^{q}(\Omega)$ function independent of $h$.

We also say that $\left\{u_{h}\right\}_{h>0}$ is bounded in $L^{q}(\Omega)$ if there exists a bounded extension $\left\{\tilde{u}_{h}\right\}_{h>0} \subset L^{q}(\Omega)$, which is equivalent to the boundedness $\left\|u_{h}\right\|_{L^{q}\left(\Omega_{h}\right)} \leq C$ for all $h>0$ and some $C>0$.

Now we have the first convergence theorem.

THEOREM 4.4. For every $h>0$ let $\bar{u}_{h}$ be a global solution of problem $\left(\mathrm{P}_{h}\right)$, then the sequence $\left\{\bar{u}_{h}\right\}_{h>0}$ is bounded in $L^{\infty}(\Omega)$ and there exist subsequences, denoted in the same way, converging to a point $\bar{u}$ in the weak $k^{\star} L^{\infty}(\Omega)$ topology. Any of these limit points is a solution of problem $(\mathrm{P})$. Moreover, we have 


$$
\lim _{h \rightarrow 0}\left\{\left\|\bar{u}-\bar{u}_{h}\right\|_{L^{\infty}\left(\Omega_{h}\right)}+\left\|\bar{\lambda}-\bar{\lambda}_{h}\right\|_{L^{\infty}\left(\Omega_{h}\right)}\right\}=0 \quad \text { and } \quad \lim _{h \rightarrow 0} J_{h}\left(\bar{u}_{h}\right)=J(\bar{u}),
$$

where $\bar{\lambda} \in \partial j(\bar{u})$ is given by (3.5c) and $\bar{\lambda}_{h} \in \partial j_{h}\left(\bar{u}_{h}\right)$ is given by $(4.4 \mathrm{c})$.

Proof. The sequence $\left\{\bar{u}_{h}\right\}_{h>0}$ is clearly bounded in $L^{\infty}(\Omega)$. Let us assume that, for a subsequence denoted in the same way, $\bar{u}_{h} \rightarrow \bar{u}$ weakly ${ }^{\star}$ in $L^{\infty}(\Omega)$ when $h \rightarrow 0$. Let $\tilde{u}$ be a solution of $(\mathrm{P})$ and take $\tilde{u}_{h} \in \mathbb{K}_{h}$ defined by

$$
\tilde{u}_{T}=\frac{1}{|T|} \int_{T} \tilde{u}(x) \mathrm{d} x \quad \forall T \in \mathcal{T}_{h} .
$$

Since $\tilde{u} \in C^{0,1}(\bar{\Omega})$ (see Corollary 3.2), we know that $\left\|\tilde{u}-\tilde{u}_{h}\right\|_{L^{\infty}\left(\Omega_{h}\right)} \rightarrow 0$. Then, using that $\bar{u} \in \mathbb{K}, \tilde{u}_{h} \in \mathbb{K}_{h}, \bar{u}_{h}$ is a solution of $\left(\mathrm{P}_{h}\right)$, and $\tilde{u}$ is a solution of $(\mathrm{P})$, we get with the help of Lemma 4.2 that

$$
J(\tilde{u}) \leq J(\bar{u}) \leq \liminf _{h \rightarrow 0} J_{h}\left(\bar{u}_{h}\right) \leq \limsup _{h \rightarrow 0} J_{h}\left(\bar{u}_{h}\right) \leq \limsup _{h \rightarrow 0} J_{h}\left(\tilde{u}_{h}\right)=J(\tilde{u}) .
$$

The above inequalities imply that $\bar{u}$ is a solution of $(\mathrm{P})$ and $J_{h}\left(\bar{u}_{h}\right) \rightarrow J(\bar{u})$. On the other hand, from Lemma 4.2 we infer

$$
\lim _{h \rightarrow 0} \int_{\Omega_{h}} L\left(x, \bar{y}_{h}\right) \mathrm{d} x=\int_{\Omega} L(x, \bar{y}) \mathrm{d} x,
$$

where $\bar{y}_{h}$ and $\bar{y}$ are the states associated with $\bar{u}_{h}$ and $\bar{u}$, respectively. Therefore

$$
\lim _{h \rightarrow 0}\left\{\frac{\nu}{2}\left\|\bar{u}_{h}\right\|_{L^{2}\left(\Omega_{h}\right)}^{2}+\mu\left\|\bar{u}_{h}\right\|_{L^{1}\left(\Omega_{h}\right)}\right\}=\frac{\nu}{2}\|\bar{u}\|_{L^{2}(\Omega)}^{2}+\mu\|\bar{u}\|_{L^{1}(\Omega)} .
$$

From this convergence and the weak convergence $\bar{u}_{h} \rightarrow \bar{u}$ (for an arbitrary extension of $\bar{u}_{h}$ to $\Omega$ ) in $L^{2}(\Omega)$ we deduce that $\bar{u}_{h} \rightarrow \bar{u}$ strongly in $L^{2}(\Omega)$. Now, Lemma 4.2 implies that $\bar{y}_{h} \rightarrow \bar{y}$ and $\bar{\varphi}_{h} \rightarrow \bar{\varphi}$ in $H^{1}(\Omega) \cap C(\bar{\Omega})$. From formula (4.4c), for every $T \in \mathcal{T}_{h}$ we deduce the existence of $x_{T} \in T$ such that

$$
\bar{\lambda}_{T}=\operatorname{Proj}_{[-1,+1]}\left(-\frac{1}{\mu|T|} \int_{T} \bar{\varphi}_{h} \mathrm{~d} x\right)=\operatorname{Proj}_{[-1,+1]}\left(-\frac{1}{\mu} \bar{\varphi}_{h}\left(x_{T}\right)\right),
$$

and therefore

$$
\begin{aligned}
\left\|\bar{\lambda}-\bar{\lambda}_{h}\right\|_{L^{\infty}\left(\Omega_{h}\right)}= & \max _{T \in \mathcal{T}_{h}}\left\|\bar{\lambda}-\bar{\lambda}_{h}\right\|_{L^{\infty}(T)} \\
\leq & \max _{T \in \mathcal{T}_{h}} \max _{x \in T}\left|\operatorname{Proj}_{[-1,+1]}\left(-\frac{1}{\mu} \bar{\varphi}(x)\right)-\operatorname{Proj}_{[-1,+1]}\left(-\frac{1}{\mu} \bar{\varphi}\left(x_{T}\right)\right)\right| \\
& +\max _{T \in \mathcal{T}_{h}}\left|\operatorname{Proj}_{[-1,+1]}\left(-\frac{1}{\mu} \bar{\varphi}\left(x_{T}\right)\right)-\operatorname{Proj}_{[-1,+1]}\left(-\frac{1}{\mu} \bar{\varphi}_{h}\left(x_{T}\right)\right)\right|,
\end{aligned}
$$

and thus

$$
\left\|\bar{\lambda}-\bar{\lambda}_{h}\right\|_{L^{\infty}\left(\Omega_{h}\right)} \leq \frac{1}{\mu} L_{\bar{\varphi}} h+\frac{1}{\mu}\left\|\bar{\varphi}-\bar{\varphi}_{h}\right\|_{L^{\infty}\left(\Omega_{h}\right)} \rightarrow 0,
$$

where $L_{\bar{\varphi}}$ is the Lipschitz constant of $\bar{\varphi}$. Finally, using (4.4a) and (3.5a), we can argue in a similar way to conclude that $\left\|\bar{u}-\bar{u}_{h}\right\|_{L^{\infty}\left(\Omega_{h}\right)} \rightarrow 0$.

The next theorem is a kind of reciprocal result to the previous one for local solutions. It is important from a practical point of view because it states that every strict local minimum of problem $(\mathrm{P})$ can be approximated by local minima of problems $\left(\mathrm{P}_{h}\right)$. 
THEOREM 4.5. Let $\bar{u}$ be a strict local minimum of $(\mathrm{P})$; then there exists a sequence $\left\{\bar{u}_{h}\right\}_{h>0}$ of local minima of problems $\left(\mathrm{P}_{h}\right)$ such that (4.8) holds.

Proof. Let $\bar{u}$ be a strict local minimum of (P); then there exists $\varepsilon>0$ such that $\bar{u}$ is the unique solution of

$$
\min _{u \in \mathbb{K} \cap \bar{B}_{\varepsilon}(\bar{u})} J(u),
$$

where $B_{\varepsilon}(\bar{u})$ is a ball in $L^{q}(\Omega)$ and all the elements of $\mathcal{U}_{h}$ are extended to $\Omega$ by taking $u_{h}(x)=\bar{u}(x)$ for any $x \in \Omega \backslash \Omega_{h}$. We will distinguish two cases: $q=2$ or $q=\infty$; recall the comments made at the beginning of section 3 . Let us consider the discrete problems

$\left(\mathrm{P}_{\varepsilon h}\right)$

$$
\min _{u \in \mathbb{K}_{h} \cap \bar{B}_{\varepsilon}(\bar{u})} J_{h}(u) .
$$

For every $h$ sufficiently small, the problem $\left(\mathrm{P}_{\varepsilon h}\right)$ has at least one solution. Indeed, the only delicate point is to check that $\mathbb{K}_{h} \cap \bar{B}_{\varepsilon}(\bar{u})$ is not empty. To this end, we define $\hat{u}_{h} \in \mathcal{U}_{h}$ by

$$
\hat{u}_{T}=\frac{1}{|T|} \int_{T} \bar{u}(x) \mathrm{d} x \quad \forall T \in \mathcal{T}_{h} .
$$

Then, thanks to the Lipschitz regularity of $\bar{u}$, we have $\left\|\bar{u}-\hat{u}_{h}\right\|_{L^{\infty}(\Omega)} \rightarrow 0$; therefore, $\hat{u} \in \mathbb{K}_{h} \cap \bar{B}_{\varepsilon}(\bar{u})$ for any $h \leq h_{0}$ and some $h_{0}>0$ sufficiently small.

Let $\bar{u}_{h}$ be a solution of $\left(\mathrm{P}_{\varepsilon h}\right)$ for $h \leq h_{0}$. Then we can argue as in the proof of Theorem 4.4 to deduce that any subsequence of $\left\{\bar{u}_{h}\right\}_{h \leq h_{0}}$ converges strongly in $L^{2}(\Omega)$ to a solution of $\left(\mathrm{P}_{\varepsilon}\right)$. Since this problem has a unique solution, we have $\left\|\bar{u}-\bar{u}_{h}\right\|_{L^{2}(\Omega)} \rightarrow 0$ for the whole sequence as $h \rightarrow 0$. If $q=2$, this implies that the constraint $\bar{u}_{h} \in \bar{B}_{\varepsilon}(\bar{u})$ is not active for $h$ small, and hence $\bar{u}_{h}$ is a local solution of $\left(\mathrm{P}_{h}\right)$ and (4.2) is fulfilled. Therefore, we proceed as in the proof of Theorem 4.4 to deduce (4.8).

If $q=\infty$, then (4.2a) and (4.2b) hold and (4.2c) has to be replaced by

$$
\int_{\Omega_{h}}\left(\bar{\varphi}_{h}+\nu \bar{u}_{h}+\mu \bar{\lambda}_{h}\right)\left(u_{h}-\bar{u}_{h}\right) \mathrm{d} x \geq 0 \quad \forall u_{h} \in \mathbb{K}_{h} \cap \bar{B}_{\varepsilon}(\bar{u}) .
$$

Let us define $\alpha_{\varepsilon}(x)=\max \{\alpha, \bar{u}(x)-\varepsilon\}, \beta_{\varepsilon}(x)=\min \{\beta, \bar{u}(x)+\varepsilon\}$, and

$$
\alpha_{\varepsilon T}=\max _{x \in T} \alpha_{\varepsilon}(x), \quad \beta_{\varepsilon T}=\min _{x \in T} \beta_{\varepsilon}(x) \quad \forall T \in \mathcal{Y}_{h}
$$

Then (4.11) is equivalent to

$$
\left(\int_{T} \bar{\varphi}_{h} \mathrm{~d} x+|T|\left[\nu \bar{u}_{T}+\mu \bar{\lambda}_{T}\right]\right)\left(u_{T}-\bar{u}_{T}\right) \geq 0 \quad \forall \alpha_{\varepsilon T} \leq u_{T} \leq \beta_{\varepsilon T}, \quad \forall T \in \mathcal{T}_{h},
$$

which leads to the representation formula analogous to (4.4a),

$$
\begin{aligned}
\bar{u}_{T} & =\operatorname{Proj}_{\left[\alpha_{\varepsilon T}, \beta_{\varepsilon T}\right]}\left(-\frac{1}{\nu}\left[\frac{1}{|T|} \int_{T} \bar{\varphi}_{h} \mathrm{~d} x+\mu \bar{\lambda}_{T}\right]\right) \\
& =\operatorname{Proj}_{\left[\alpha_{\varepsilon T}, \beta_{\varepsilon T}\right]}\left(-\frac{1}{\nu}\left[\bar{\varphi}_{h}\left(x_{T}\right)+\mu \bar{\lambda}_{T}\right]\right) \quad \forall T \in \mathcal{T}_{h} .
\end{aligned}
$$

Copyright $\odot$ by SIAM. Unauthorized reproduction of this article is prohibited. 
The representation formula (4.4c) is still valid, which leads to the convergence $\| \bar{\lambda}-$ $\bar{\lambda}_{h} \|_{L^{\infty}\left(\Omega_{h}\right)} \rightarrow 0$ as proved in Theorem 4.4. On the other hand, it is obvious that

$$
\operatorname{Proj}_{[\alpha, \beta]}\left(-\frac{1}{\nu}[\bar{\varphi}(x)+\mu \bar{\lambda}(x)]\right)=\operatorname{Proj}_{\left[\alpha_{\varepsilon}(x), \beta_{\varepsilon}(x)\right]}\left(-\frac{1}{\nu}[\bar{\varphi}(x)+\mu \bar{\lambda}(x)]\right) \quad \forall x \in \Omega .
$$

Also we have

$$
\left|\alpha_{\varepsilon}(x)-\alpha_{\varepsilon T}\right| \leq \max _{x \in T} \bar{u}(x)-\min _{x \in T} \bar{u}(x) \leq L_{\bar{u}} h \quad \forall x \in T, \quad \forall T \in \mathcal{T}_{h},
$$

where $L_{\bar{u}}$ is the Lipschitz constant of $\bar{u}$. An analogous inequality is valid for $\beta_{\varepsilon}(x)-$ $\beta_{\varepsilon T}$. Finally, we have for any $x \in T$, with $T \in \mathcal{T}_{h}$,

$$
\begin{aligned}
\left|\bar{u}(x)-\bar{u}_{h}(x)\right| \leq \mid \bar{u}(x)- & \bar{u}\left(x_{T}\right)|+| \bar{u}\left(x_{T}\right)-\bar{u}_{T} \mid \\
\leq & L_{\bar{u}} h+\mid \operatorname{Proj}_{\left[\alpha_{\varepsilon}\left(x_{T}\right), \beta_{\varepsilon}\left(x_{T}\right)\right]}\left(-\frac{1}{\nu}\left[\bar{\varphi}\left(x_{T}\right)+\mu \bar{\lambda}\left(x_{T}\right)\right]\right) \\
& \quad-\operatorname{Proj}_{\left[\alpha_{\varepsilon T}, \beta_{\varepsilon T}\right]}\left(-\frac{1}{\nu}\left[\bar{\varphi}_{h}\left(x_{T}\right)+\mu \bar{\lambda}_{T}\right]\right) \mid \\
\leq & L_{\bar{u}} h+\left|\alpha_{\varepsilon}\left(x_{T}\right)-\alpha_{\varepsilon T}\right|+\left|\beta_{\varepsilon}\left(x_{T}\right)-\beta_{\varepsilon T}\right| \\
& \quad+\frac{1}{\nu}\left\{\left|\bar{\varphi}\left(x_{T}\right)-\bar{\varphi}_{h}\left(x_{T}\right)\right|+\mu\left|\bar{\lambda}\left(x_{T}\right)-\bar{\lambda}_{T}\right|\right\} \\
\leq 3 L_{\bar{u}} h & +\frac{1}{\nu}\left\{\left\|\bar{\varphi}-\bar{\varphi}_{h}\right\|_{L^{\infty}\left(\Omega_{h}\right)}+\mu\left\|\bar{\lambda}-\bar{\lambda}_{h}\right\|_{L^{\infty}\left(\Omega_{h}\right)}\right\} \rightarrow 0 .
\end{aligned}
$$

Hence, we have that $\left\|\bar{u}-\bar{u}_{h}\right\|_{L^{\infty}\left(\Omega_{h}\right)} \rightarrow 0$ as $h \rightarrow 0$; therefore, the constraint $\bar{u}_{h} \in$ $\bar{B}_{\varepsilon}(\bar{u})$ is not active for small $h$. Consequently $\bar{u}$ is a local minimum of $\left(\mathrm{P}_{h}\right)$.

4.2. Error estimates. In this section, $\left\{\bar{u}_{h}\right\}_{h>0}$ denotes a sequence of local minima of problems $\left(\mathrm{P}_{h}\right)$ such that $\left\|\bar{u}-\bar{u}_{h}\right\|_{L^{\infty}\left(\Omega_{h}\right)} \rightarrow 0$ when $h \rightarrow 0, \bar{u}$ being a local minimum of $(\mathrm{P})$; see Theorems 4.4 and 4.5. The goal of this section is to obtain estimates of $\bar{u}-\bar{u}_{h}$ in the $L^{2}$ and $L^{\infty}$ norms. As we did in the proof of Theorem 4.5, we extend all the functions $u_{h} \in \mathcal{U}_{h}$ to $\Omega$ by taking $u_{h}(x)=\bar{u}(x)$ for every $x \in \Omega \backslash \Omega_{h}$. Analogously we extend $\bar{\lambda}_{h}$ to $\Omega$ by setting $\bar{\lambda}_{h}(x)=\bar{\lambda}(x)$ for $x \in \Omega \backslash \Omega_{h}$. Now, we recall that Corollary 3.2 implies that $\bar{u}, \bar{\lambda} \in C^{0,1}(\bar{\Omega})$, where $\bar{\lambda} \in \partial j(\bar{u})$ and $(\bar{u}, \bar{\lambda})$ satisfies (3.4) along with the state $\bar{y}$ and the adjoint state $\bar{\varphi}$ associated with $\bar{u}$.

To derive the error estimates we are going to begin by invoking the first-order optimality conditions (3.4c) and (4.2c). Taking $u=\bar{u}_{h}$ in (3.4c), we get

$$
F^{\prime}(\bar{u})\left(\bar{u}_{h}-\bar{u}\right)+\mu \int_{\Omega} \bar{\lambda}\left(\bar{u}_{h}-\bar{u}\right) \mathrm{d} x=\int_{\Omega}(\bar{\varphi}+\nu \bar{u}+\mu \bar{\lambda})\left(\bar{u}_{h}-\bar{u}\right) \mathrm{d} x \geq 0 .
$$

Now, for any $u_{h} \in \mathbb{K}_{h}$, we deduce from (4.2c) that

$$
F_{h}^{\prime}\left(\bar{u}_{h}\right)\left(u_{h}-\bar{u}_{h}\right)+\mu \int_{\Omega} \bar{\lambda}_{h}\left(u_{h}-\bar{u}_{h}\right) \mathrm{d} x=\int_{\Omega_{h}}\left(\bar{\varphi}_{h}+\nu \bar{u}_{h}+\mu \bar{\lambda}_{h}\right)\left(u_{h}-\bar{u}_{h}\right) \mathrm{d} x \geq 0 .
$$

From here we get

$$
\begin{array}{r}
F^{\prime}\left(\bar{u}_{h}\right)\left(\bar{u}-\bar{u}_{h}\right)+\left[F_{h}^{\prime}\left(\bar{u}_{h}\right)-F^{\prime}\left(\bar{u}_{h}\right)\right]\left(\bar{u}-\bar{u}_{h}\right)+\left[F_{h}^{\prime}\left(\bar{u}_{h}\right)-F^{\prime}(\bar{u})\right]\left(u_{h}-\bar{u}\right) \\
+F^{\prime}(\bar{u})\left(u_{h}-\bar{u}\right)+\mu \int_{\Omega_{h}} \bar{\lambda}_{h}\left(u_{h}-\bar{u}_{h}\right) \mathrm{d} x \geq 0 .
\end{array}
$$

Copyright $@$ by SIAM. Unauthorized reproduction of this article is prohibited. 
Adding (4.12) and (4.13) we deduce that

$$
\begin{aligned}
& {\left[F^{\prime}\left(\bar{u}_{h}\right)-F^{\prime}(\bar{u})\right]\left(\bar{u}_{h}-\bar{u}\right) \leq\left[F_{h}^{\prime}\left(\bar{u}_{h}\right)-F^{\prime}\left(\bar{u}_{h}\right)\right]\left(\bar{u}-\bar{u}_{h}\right)} \\
& \quad+\left[F_{h}^{\prime}\left(\bar{u}_{h}\right)-F^{\prime}(\bar{u})\right]\left(u_{h}-\bar{u}\right)+F^{\prime}(\bar{u})\left(u_{h}-\bar{u}\right)+\mu \int_{\Omega} \bar{\lambda}\left(u_{h}-\bar{u}\right) \mathrm{d} x \\
& \quad+\mu \int_{\Omega}\left(\bar{\lambda}-\bar{\lambda}_{h}\right)\left(\bar{u}_{h}-\bar{u}\right) \mathrm{d} x+\mu \int_{\Omega}\left(\bar{\lambda}_{h}-\bar{\lambda}\right)\left(u_{h}-\bar{u}\right) \mathrm{d} x
\end{aligned}
$$

for any $u_{h} \in \mathbb{K}_{h}$. This inequality is crucial in the proof of error estimates. To deal with the left-hand side of (4.14) we need $\bar{u}$ to satisfy the sufficient second-order condition $F^{\prime \prime}(\bar{u}) v^{2}>0$ for every $v \in C_{\bar{u}} \backslash\{0\}$, or, equivalently (see Theorem 3.8),

$$
\exists \delta>0 \text { and } \exists \tau>0 \text { such that } F^{\prime \prime}(\bar{u}) v^{2} \geq \delta\|v\|_{L^{2}(\Omega)}^{2} \quad \forall v \in C_{\bar{u}}^{\tau} .
$$

Lemma 4.6. Let us assume that (4.15) holds. Then, there exists $h_{\delta}>0$ such that

$$
\frac{\delta}{2}\left\|\bar{u}-\bar{u}_{h}\right\|_{L^{2}\left(\Omega_{h}\right)}^{2} \leq\left[F^{\prime}\left(\bar{u}_{h}\right)-F^{\prime}(\bar{u})\right]\left(\bar{u}_{h}-\bar{u}\right) \quad \forall h \leq h_{\delta} .
$$

Proof. Using the mean value theorem we obtain

$$
\left[F^{\prime}\left(\bar{u}_{h}\right)-F^{\prime}(\bar{u})\right]\left(\bar{u}_{h}-\bar{u}\right)=F^{\prime \prime}\left(\bar{u}+\theta_{h}\left(\bar{u}_{h}-\bar{u}\right)\right)\left(\bar{u}_{h}-\bar{u}\right)^{2} .
$$

On the other hand, since $F$ is of class $C^{2}$ in $L^{2}(\Omega)$, there exists $\varepsilon>0$ such that

$$
\left|\left[F^{\prime \prime}(\bar{u})-F^{\prime \prime}(v)\right]\left(\bar{u}_{h}-\bar{u}\right)^{2}\right| \leq \frac{\delta}{2}\left\|\bar{u}_{h}-\bar{u}\right\|_{L^{2}\left(\Omega_{h}\right)}^{2} \quad \text { if } \quad\|\bar{u}-v\|_{L^{2}(\Omega)}<\varepsilon .
$$

From the convergence $\left\|\bar{u}-\bar{u}_{h}\right\|_{L^{\infty}\left(\Omega_{h}\right)} \rightarrow 0$, we deduce the existence of $h_{\varepsilon}>0$ such that $\left\|\bar{u}-\bar{u}_{h}\right\|_{L^{2}(\Omega)}<\varepsilon$ for $h \leq h_{\varepsilon}$. Then, the last two relations lead to

$$
\begin{aligned}
{\left[F^{\prime}\left(\bar{u}_{h}\right)-F^{\prime}(\bar{u})\right] } & \left(\bar{u}_{h}-\bar{u}\right) \\
& \geq F^{\prime \prime}(\bar{u})\left(\bar{u}_{h}-\bar{u}\right)^{2}-\left|\left[F^{\prime \prime}\left(\bar{u}+\theta_{h}\left(\bar{u}_{h}-\bar{u}\right)\right)-F^{\prime \prime}(\bar{u})\right]\left(\bar{u}_{h}-\bar{u}\right)^{2}\right| \\
& \geq F^{\prime \prime}(\bar{u})\left(\bar{u}_{h}-\bar{u}\right)^{2}-\frac{\delta}{2}\left\|\bar{u}_{h}-\bar{u}\right\|_{L^{2}\left(\Omega_{h}\right)}^{2} \quad \forall h \leq h_{\varepsilon} .
\end{aligned}
$$

If we prove that $\bar{u}_{h}-\bar{u} \in C_{\bar{u}}^{\tau}$ for every $h$ small enough, then (4.16) follows from (4.15) and the previous inequality. Therefore, the rest of the proof is devoted to showing that $\bar{u}_{h}-\bar{u} \in C_{\bar{u}}^{\tau}$ for every $h$ sufficiently small. Let us define

$$
v_{h}=\frac{\bar{u}_{h}-\bar{u}}{\left\|\bar{u}_{h}-\bar{u}\right\|_{L^{2}(\Omega)}}
$$

then there exist an element $v \in L^{2}(\Omega)$ and a sequence $h_{k} \rightarrow 0$ such that $v_{h_{k}} \rightarrow v$ in $L^{2}(\Omega)$. It is obvious that each $v_{h}$ satisfies (3.7), and thus $v$ also does. On the other hand, (4.8) and Lemma 4.2 imply that $\left\|\bar{d}-\bar{d}_{h}\right\|_{L^{\infty}\left(\Omega_{h}\right)} \rightarrow 0$, where $\bar{d}$ and $\bar{d}_{h}$ are defined by (3.10) and (4.5), respectively. From (3.11), we know that $\bar{u}\left(x_{0}\right)=\alpha$ whenever $\bar{d}\left(x_{0}\right)>0$. Moreover, there exist $\rho>0$ and $h_{\rho}>0$ such that $\bar{d}_{h}(x)>0$ for almost all $x \in \Omega$ satisfying $\left|x-x_{0}\right|<\rho$ and $h \leq h_{\rho}<\rho$. Then, (4.6) implies that $\bar{u}_{h}\left(x_{0}\right)=\alpha$ too; hence $v_{h}\left(x_{0}\right)=0$ for $h \leq h_{\rho}$ and almost all $x_{0}$ satisfying $\bar{d}\left(x_{0}\right)>0$. Analogously, we can prove that $v_{h}\left(x_{0}\right)=0$ for $h$ small enough if $\bar{d}\left(x_{0}\right)<0$. We have

Copyright $@$ by SIAM. Unauthorized reproduction of this article is prohibited. 
that $v_{h_{k}} \rightarrow v$ in $L^{2}\left(\Omega_{0}\right)$, where $\Omega_{0}=\{x \in \Omega: \bar{d}(x) \neq 0\}$. And also we have $v_{h_{k}}(x) \rightarrow 0$ pointwise for almost every $x \in \Omega_{0}$. Consequently, $v=0$ in $\Omega_{0}$ holds (see [12, p. 207]), and therefore

$$
F^{\prime}(\bar{u}) v+\mu \int_{\Omega} \bar{\lambda} v \mathrm{~d} x=\int_{\Omega} \bar{d} v \mathrm{~d} x=\int_{\Omega}|\bar{d}||v| \mathrm{d} x=0 .
$$

Now, we study the limit of $j^{\prime}\left(\bar{u} ; v_{h_{k}}\right)$. First we observe that

$$
\lim _{k \rightarrow \infty}\left\{\int_{\Omega_{\bar{u}}^{+}} v_{h_{k}} \mathrm{~d} x-\int_{\Omega_{\overline{\bar{u}}}^{-}} v_{h_{k}} \mathrm{~d} x\right\}=\int_{\Omega_{\bar{u}}^{+}} v \mathrm{~d} x-\int_{\Omega_{\overline{\bar{u}}}^{-}} v \mathrm{~d} x .
$$

The limit in the integral over $\Omega_{\bar{u}}^{0}$ is more complicated. First, we observe that (3.5b) implies

$$
\Omega_{\bar{u}}^{0}=\{x \in \Omega:|\bar{\varphi}(x)| \leq \mu\} .
$$

If $\left|\bar{\varphi}\left(x_{0}\right)\right|<\mu$, then arguing as above we have that $\left|\bar{\varphi}_{h}(x)\right|<\mu$ for $\left|x-x_{0}\right|<\rho$ and $h \leq h_{\rho}<\rho$; hence (4.4b) implies that $\bar{u}_{h}\left(x_{0}\right)=0$ for $h \leq h_{\rho}$. Thus, we have that $v_{h_{k}}(x) \rightarrow 0$ for $|\bar{\varphi}(x)|<\mu$, which leads to $v(x)=0$ in the same set.

If $\bar{\varphi}\left(x_{0}\right)=\mu$, then (3.5c) implies that $\bar{\lambda}\left(x_{0}\right)=-1$. Consequently $\bar{\lambda}_{h}(x)<0$ in a neighborhood of $x_{0}$ for every $h$ small; hence with (4.3) we get that $\bar{u}_{h}\left(x_{0}\right) \leq 0$ for every small $h$. Thus $v_{h_{k}}(x) \leq 0$ whenever $\bar{\varphi}(x)=\mu$ and $h$ is small, and hence $v(x) \leq 0$ in the same set. Analogously we obtain that $v_{h_{k}}(x) \geq 0$ whenever $\bar{\varphi}(x)=-\mu$ and $h$ is small, and consequently $v(x) \geq 0$. These results lead to

$$
\begin{aligned}
& \lim _{k \rightarrow \infty} \int_{\Omega_{u}^{0}}\left|v_{h_{k}}\right| \mathrm{d} x=\lim _{k \rightarrow \infty}\left\{\int_{\Omega_{\mu}^{+}}\left|v_{h_{k}}\right| \mathrm{d} x+\int_{\Omega_{\mu}^{-}}\left|v_{h_{k}}\right| \mathrm{d} x\right\} \\
= & \lim _{k \rightarrow \infty}\left\{-\int_{\Omega_{\mu}^{+}} v_{h_{k}} \mathrm{~d} x+\int_{\Omega_{\mu}^{-}} v_{h_{k}} \mathrm{~d} x\right\}=-\int_{\Omega_{\mu}^{+}} v \mathrm{~d} x+\int_{\Omega_{\mu}^{-}} v \mathrm{~d} x=\int_{\Omega_{u}^{0}}|v| \mathrm{d} x,
\end{aligned}
$$

where

$$
\Omega_{\mu}^{+}=\left\{x \in \Omega_{\bar{u}}^{0}: \bar{\varphi}(x)=+\mu\right\} \text { and } \Omega_{\mu}^{-}=\left\{x \in \Omega_{\bar{u}}^{0}: \bar{\varphi}(x)=-\mu\right\} .
$$

From (4.18), (4.19), and the fact that $\bar{\lambda}(x)=+1$ (respectively, -1 ) for $x \in \Omega_{\mu}^{-}$ (respectively, $\Omega_{\mu}^{+}$), we deduce that

$$
\lim _{k \rightarrow \infty} j^{\prime}\left(\bar{u} ; v_{h_{k}}\right)=j^{\prime}(\bar{u} ; v)=\int_{\Omega} \bar{\lambda} v \mathrm{~d} x .
$$

From identities (4.17) and (4.20) it follows that

$$
\lim _{k \rightarrow \infty}\left\{F^{\prime}(\bar{u}) v_{h_{k}}+\mu j^{\prime}\left(\bar{u} ; v_{h_{k}}\right)\right\}=F^{\prime}(\bar{u}) v+\mu j^{\prime}(\bar{u} ; v)=0 .
$$

This equality holds for any weakly convergent subsequence of $\left\{v_{h}\right\}_{h>0}$; therefore

$$
\lim _{h \rightarrow 0}\left\{F^{\prime}(\bar{u}) v_{h}+\mu j^{\prime}\left(\bar{u} ; v_{h}\right)\right\}=0 .
$$

Consequently, given $\tau>0$ satisfying (4.15), there exists $h_{\tau}>0$ such that

$$
F^{\prime}(\bar{u}) v_{h}+\mu j^{\prime}\left(\bar{u} ; v_{h}\right) \leq \tau \quad \forall h \leq h_{\tau}
$$

Copyright $@$ by SIAM. Unauthorized reproduction of this article is prohibited. 
From this inequality and the definition of $v_{h}$ we conclude that

$$
F^{\prime}(\bar{u})\left(\bar{u}_{h}-\bar{u}\right)+\mu j^{\prime}\left(\bar{u} ; \bar{u}_{h}-\bar{u}\right) \leq \tau\left\|\bar{u}_{h}-\bar{u}\right\|_{L^{2}(\Omega)},
$$

which concludes the proof of $\bar{u}_{h}-\bar{u} \in C_{\bar{u}}^{\tau}$. Thus, inequality (4.16) holds for any $h \leq h_{\delta}=\min \left\{h_{\varepsilon}, h_{\tau}\right\}$.

Combining (4.14) and (4.16), and assuming that $\bar{u}$ satisfies the second-order sufficient condition (4.15), we get

$$
\begin{aligned}
& \frac{\delta}{2}\left\|\bar{u}-\bar{u}_{h}\right\|_{L^{2}\left(\Omega_{h}\right)}^{2} \leq {\left[F_{h}^{\prime}\left(\bar{u}_{h}\right)-F^{\prime}\left(\bar{u}_{h}\right)\right]\left(\bar{u}-\bar{u}_{h}\right) } \\
&+\left[F_{h}^{\prime}\left(\bar{u}_{h}\right)-F^{\prime}(\bar{u})\right]\left(u_{h}-\bar{u}\right)+F^{\prime}(\bar{u})\left(u_{h}-\bar{u}\right)+\mu \int_{\Omega} \bar{\lambda}\left(u_{h}-\bar{u}\right) \mathrm{d} x \\
& \quad+\mu \int_{\Omega}\left(\bar{\lambda}-\bar{\lambda}_{h}\right)\left(\bar{u}_{h}-\bar{u}\right) \mathrm{d} x+\mu \int_{\Omega}\left(\bar{\lambda}_{h}-\bar{\lambda}\right)\left(u_{h}-\bar{u}\right) \mathrm{d} x
\end{aligned}
$$

for any $u_{h} \in \mathbb{K}_{h}$. The rest of the section is devoted to estimating the right-hand side of the above inequality. To deal with the first two terms we give the following lemma.

Lemma 4.7. Let $u, v \in L^{\infty}(\Omega)$ be bounded by a constant $M>0$ and let $w \in$ $L^{2}(\Omega)$, with $w(x)=0$ in $\Omega \backslash \Omega_{h}$. Then, there exists $C_{M}>0$ such that

$$
\left|\left[F_{h}^{\prime}(v)-F^{\prime}(u)\right] w\right| \leq C_{M}\left(h^{2}+\|u-v\|_{L^{2}(\Omega)}\right)\|w\|_{L^{2}(\Omega)} .
$$

Proof. If we denote by $\varphi_{h}(v)$ and $\varphi_{u}$ the discrete and continuous adjoint states associated with $v$ and $u$, respectively, we have

$$
\begin{aligned}
\left|\left[F_{h}^{\prime}(v)-F^{\prime}(u)\right] w\right| & =\left|\int_{\Omega}\left(\varphi_{h}(v)+\nu v\right) w \mathrm{~d} x-\int_{\Omega}\left(\varphi_{u}+\nu u\right) w \mathrm{~d} x\right| \\
& \leq\left(\left\|\varphi_{h}(v)-\varphi_{u}\right\|_{L^{2}(\Omega)}+\nu\|v-u\|_{L^{2}(\Omega)}\right)\|w\|_{L^{2}(\Omega)} .
\end{aligned}
$$

Now, it is enough to use (4.7b) to deduce (4.22) from the above inequality.

Using (4.22) and Young's inequality we can estimate the first two terms in (4.21) by

$$
\begin{aligned}
& {\left[F_{h}^{\prime}\left(\bar{u}_{h}\right)-F^{\prime}\left(\bar{u}_{h}\right)\right]\left(\bar{u}-\bar{u}_{h}\right)+\left[F_{h}^{\prime}\left(\bar{u}_{h}\right)-F^{\prime}(\bar{u})\right]\left(u_{h}-\bar{u}\right)} \\
& \leq C\left\{h^{2}\left\|\bar{u}_{h}-\bar{u}\right\|_{L^{2}\left(\Omega_{h}\right)}+\left[h^{2}+\left\|\bar{u}_{h}-\bar{u}\right\|_{L^{2}\left(\Omega_{h}\right)}\right]\left\|u_{h}-\bar{u}\right\|_{L^{2}\left(\Omega_{h}\right)}\right\} \\
& \leq \frac{\delta}{4}\left\|\bar{u}_{h}-\bar{u}\right\|_{L^{2}\left(\Omega_{h}\right)}^{2}+C^{\prime}\left\{h^{4}+\left\|u_{h}-\bar{u}\right\|_{L^{2}\left(\Omega_{h}\right)}^{2}\right\} .
\end{aligned}
$$

From this inequality and (4.21) we infer

$$
\begin{aligned}
\frac{\delta}{4}\left\|\bar{u}-\bar{u}_{h}\right\|_{L^{2}\left(\Omega_{h}\right)}^{2} \leq & C^{\prime}\left\{h^{4}+\left\|u_{h}-\bar{u}\right\|_{L^{2}\left(\Omega_{h}\right)}^{2}\right\}+F^{\prime}(\bar{u})\left(u_{h}-\bar{u}\right)+\mu \int_{\Omega} \bar{\lambda}\left(u_{h}-\bar{u}\right) \mathrm{d} x \\
& +\mu \int_{\Omega}\left(\bar{\lambda}-\bar{\lambda}_{h}\right)\left(\bar{u}_{h}-\bar{u}\right) \mathrm{d} x+\mu \int_{\Omega}\left(\bar{\lambda}_{h}-\bar{\lambda}\right)\left(u_{h}-\bar{u}\right) \mathrm{d} x
\end{aligned}
$$

for any $u_{h} \in \mathbb{K}_{h}$. Let us introduce a convenient element $\tilde{u}_{h} \in \mathbb{K}_{h}$ which approximates $\bar{u}$. We define

$$
\tilde{u}_{h}=\sum_{T \in \mathcal{T}_{h}} \tilde{u}_{T} \chi_{T}, \quad \text { where } \quad \tilde{u}_{T}= \begin{cases}\frac{1}{I_{T}} \int_{T} \bar{u}(x) \mathrm{d} x & \text { if } I_{T} \neq 0 \\ \frac{1}{|T|} \int_{T} \bar{u}(x) \mathrm{d} x & \text { otherwise }\end{cases}
$$

Copyright $@$ by SIAM. Unauthorized reproduction of this article is prohibited. 
with

$$
I_{T}=\int_{T} \bar{d}(x) \mathrm{d} x
$$

Now $\tilde{u}_{h}$ is extended to $\Omega$ by setting $\tilde{u}_{h}(x)=\bar{u}(x)$ for $x \in \Omega \backslash \Omega_{h}$.

LEMMA 4.8. The following statements hold:

1. $\tilde{u}_{h} \in \mathbb{K}_{h}$,

2. $\left\|\tilde{u}_{h}-\bar{u}\right\|_{L^{\infty}\left(\Omega_{h}\right)} \leq C h$,

3. $F^{\prime}(\bar{u})\left(\tilde{u}_{h}-\bar{u}\right)+\mu \int_{\Omega} \bar{\lambda}\left(\tilde{u}_{h}-\bar{u}\right) \mathrm{d} x=\int_{\Omega} \bar{d}\left(\tilde{u}_{h}-\bar{u}\right) \mathrm{d} x=0$.

The proof of this lemma follows the steps of [6, Lemma 4.8]. Indeed, $\bar{\lambda}$ does not play any role in the proof. The only thing to take into account is that $\bar{u}$ and $\bar{d}$ are Lipschitz functions. Inserting this control $\tilde{u}_{h}$ into (4.23) we get

$$
\frac{\delta}{4}\left\|\bar{u}-\bar{u}_{h}\right\|_{L^{2}\left(\Omega_{h}\right)}^{2} \leq C\left[h^{2}+h\left\|\bar{\lambda}_{h}-\bar{\lambda}\right\|_{L^{2}\left(\Omega_{h}\right)}\right]+\mu \int_{\Omega}\left(\bar{\lambda}-\bar{\lambda}_{h}\right)\left(\bar{u}_{h}-\bar{u}\right) \mathrm{d} x .
$$

Let us estimate $\bar{\lambda}-\bar{\lambda}_{h}$. By the estimates (4.9) and (4.7c) we infer

$$
\left\|\bar{\lambda}-\bar{\lambda}_{h}\right\|_{L^{\infty}\left(\Omega_{h}\right)} \leq C\left[h+\left\|\bar{u}_{h}-\bar{u}\right\|_{L^{2}\left(\Omega_{h}\right)}\right] .
$$

Inserting this estimate into (4.24) and using once again Young's inequality we get

$$
\frac{\delta}{8}\left\|\bar{u}-\bar{u}_{h}\right\|_{L^{2}\left(\Omega_{h}\right)}^{2} \leq C h^{2}+\mu \int_{\Omega}\left(\bar{\lambda}-\bar{\lambda}_{h}\right)\left(\bar{u}_{h}-\bar{u}\right) \mathrm{d} x .
$$

Finally, we estimate the last term.

LEMMA 4.9. The following inequality holds:

$$
\int_{\Omega}\left(\bar{\lambda}-\bar{\lambda}_{h}\right)\left(\bar{u}_{h}-\bar{u}\right) \mathrm{d} x \leq 0
$$

Proof. By (3.1), (4.3), and the property $\bar{u}_{h}=\bar{u}$ in $\Omega \backslash \Omega_{h}$, we get

$$
\begin{aligned}
\int_{\Omega}\left(\bar{\lambda}-\bar{\lambda}_{h}\right)\left(\bar{u}_{h}-\bar{u}\right) \mathrm{d} x & =\int_{\Omega} \bar{\lambda}\left(\bar{u}_{h}-\bar{u}\right) \mathrm{d} x+\int_{\Omega_{h}} \bar{\lambda}_{h}\left(\bar{u}-\bar{u}_{h}\right) \mathrm{d} x \\
& \leq\left\|\bar{u}_{h}\right\|_{L^{1}(\Omega)}-\|\bar{u}\|_{L^{1}(\Omega)}+\|\bar{u}\|_{L^{1}\left(\Omega_{h}\right)}-\left\|\bar{u}_{h}\right\|_{L^{1}\left(\Omega_{h}\right)}=0 .
\end{aligned}
$$

With (4.25) and (4.26) we have proved the following theorem.

THEOREM 4.10. Let $\bar{u}$ be a local minimum of problem $(\mathrm{P})$ and let $\left\{\bar{u}_{h}\right\}_{h>0}$ be a sequence of local minima of problems $\left(\mathrm{P}_{h}\right)$ such that $\left\|\bar{u}-\bar{u}_{h}\right\|_{L^{\infty}\left(\Omega_{h}\right)} \rightarrow 0$. Let us assume that (4.15) holds. Then there exists a constant $C>0$ independent of $h$ such that $\left\|\bar{u}_{h}-\bar{u}\right\|_{L^{2}\left(\Omega_{h}\right)} \leq C h$.

Finally, combining this theorem with (4.7c) and (4.9) and the representation formulas for $\bar{u}$ and $\bar{u}_{h}$ we get the following result.

COROLlary 4.11. Under the assumptions of Theorem 4.10, we have

$$
\left\|\bar{u}_{h}-\bar{u}\right\|_{L^{\infty}\left(\Omega_{h}\right)}+\left\|\bar{\lambda}_{h}-\bar{\lambda}\right\|_{L^{\infty}\left(\Omega_{h}\right)}+\left\|\bar{y}_{h}-\bar{y}\right\|_{L^{\infty}\left(\Omega_{h}\right)}+\left\|\bar{\varphi}_{h}-\bar{\varphi}\right\|_{L^{\infty}\left(\Omega_{h}\right)} \leq C h,
$$

for some constant $C>0$ independent of $h$.

Copyright $@$ by SIAM. Unauthorized reproduction of this article is prohibited. 
5. A variational discretization of $(\mathbf{P})$. In this section we consider a partial discretization of $(\mathrm{P})$. As in section 4, we consider a triangulation of $\Omega$ under the same hypotheses; $Y_{h}$ is defined in the same way leading to the same discrete state equation (4.1). However, we do not discretize the controls and we set $\mathcal{U}_{h}=L^{\infty}(\Omega)$. Rather, the controls are implicitly discretized by the representation formula; see (5.1). This idea was introduced by Hinze [13] and was called variational discretization of the control problem. This discretization is numerically implementable (although the discretized problem continues to be an infinite dimensional optimization problem) thanks to the fact that the optimal control $\bar{u}_{h}$ is a projection of the adjoint state, which is piecewise linear, translating this property to $\bar{u}_{h}$. This incomplete discretization leads to an error estimate of $\bar{u}-\bar{u}_{h}$ of order $h^{2}$ in the $L^{2}\left(\Omega_{h}\right)$ norm as we will prove in this section.

The problem $\left(\mathrm{Q}_{h}\right)$ is defined as follows:

$$
\left\{\begin{array}{l}
\min J_{h}\left(u_{h}\right)=F_{h}\left(u_{h}\right)+\mu j_{h}\left(u_{h}\right), \\
u_{h} \in \mathbb{K},
\end{array}\right.
$$

where $F_{h}: L^{2}\left(\Omega_{h}\right) \longrightarrow \mathbb{R}$ and $j_{h}: L^{1}\left(\Omega_{h}\right) \longrightarrow \mathbb{R}$ are defined as in section 4 . The proof of the existence of a solution $\bar{u}_{h}$ of $\left(\mathrm{Q}_{h}\right)$ is the same as for the problem $(\mathrm{P})$. The optimality conditions satisfied by a local minimum of $\left(\mathrm{Q}_{h}\right)$ are given by Theorem 4.1 with $\mathbb{K}_{h}$ replaced by $\mathbb{K}$. This change leads to the same relations formulas proved in Corollary 3.2, i.e.,

$$
\begin{aligned}
& \bar{u}_{h}(x)=\operatorname{Proj}_{[\alpha, \beta]}\left(-\frac{1}{\nu}\left(\bar{\varphi}_{h}(x)+\mu \bar{\lambda}_{h}(x)\right)\right), \\
& \bar{u}_{h}(x)=0 \Leftrightarrow\left|\bar{\varphi}_{h}(x)\right| \leq \mu, \\
& \bar{\lambda}_{h}(x)=\operatorname{Proj}_{[-1,+1]}\left(-\frac{1}{\mu} \bar{\varphi}_{h}(x)\right) .
\end{aligned}
$$

These expressions are valid for every $x \in \Omega_{h}$. Also we have

$$
\begin{cases}\bar{\lambda}_{h}(x)=+1 & \text { if } \bar{u}_{h}(x)>0, \\ \bar{\lambda}_{h}(x)=-1 & \text { if } \bar{u}_{h}(x)<0, \\ \bar{\lambda}_{h}(x) \in[-1,+1] & \text { if } \bar{u}_{h}(x)=0 .\end{cases}
$$

Theorem 4.4 is valid for the problem $\left(\mathrm{Q}_{h}\right)$. Let us mention the only two changes in the proof. First, given a solution $\tilde{u}$ of $(\mathrm{P})$, we do not need to introduce $\tilde{u}_{h}$ as we did in the proof; we just take $\tilde{u}_{h}=\tilde{u}$ because now $\mathbb{K}_{h}=\mathbb{K}$. On the other hand, using (3.5c) and (5.1c) we have that

$$
\left\|\bar{\lambda}-\bar{\lambda}_{h}\right\|_{L^{\infty}\left(\Omega_{h}\right)}=\left\|\operatorname{Proj}_{[\alpha, \beta]}\left(-\frac{1}{\mu} \bar{\varphi}\right)-\operatorname{Proj}_{[\alpha, \beta]}\left(-\frac{1}{\mu} \bar{\varphi}_{h}\right)\right\|_{L^{\infty}\left(\Omega_{h}\right)} \leq \frac{1}{\mu}\left\|\bar{\varphi}-\bar{\varphi}_{h}\right\|_{L^{\infty}\left(\Omega_{h}\right)}
$$

and

$$
\left\|\bar{u}-\bar{u}_{h}\right\|_{L^{\infty}\left(\Omega_{h}\right)} \leq \frac{1}{\nu}\left\|(\bar{\varphi}+\mu \bar{\lambda})-\left(\bar{\varphi}_{h}+\mu \bar{\lambda}_{h}\right)\right\|_{L^{\infty}\left(\Omega_{h}\right)} .
$$

With these changes the proof follows the same steps. Theorem 4.5 is also valid. In fact, its proof is easier for the new problem $\left(\mathrm{Q}_{h}\right)$ by using the properties (5.1). For instance, in the definition of $\left(\mathrm{P}_{\varepsilon}\right)$ we have to replace $\mathbb{K}_{h}$ by $\mathbb{K}$; then it is obvious that 
$\bar{u}$ is a feasible control of $\left(\mathrm{P}_{\varepsilon}\right)$. We consider the functions $\alpha_{\varepsilon}$ and $\beta_{\varepsilon}$ and we get from (4.11) that

$$
\bar{u}_{h}(x)=\operatorname{Proj}_{\left[\alpha_{\varepsilon}, \beta_{\varepsilon}\right]}\left(-\frac{1}{\nu}\left[\bar{\varphi}_{h}(x)+\mu \bar{\lambda}_{h}(x)\right]\right) .
$$

Moreover, from the definition of $\alpha_{\varepsilon}$ and $\beta_{\varepsilon}$ we also have

$$
\bar{u}(x)=\operatorname{Proj}_{\left[\alpha_{\varepsilon}, \beta_{\varepsilon}\right]}\left(-\frac{1}{\nu}[\bar{\varphi}(x)+\mu \bar{\lambda}(x)]\right) .
$$

From the last two inequalities we deduce that

$$
\left\|\bar{u}-\bar{u}_{h}\right\|_{L^{\infty}\left(\Omega_{h}\right)} \leq \frac{1}{\nu}\left\|(\bar{\varphi}+\mu \bar{\lambda})-\left(\bar{\varphi}_{h}+\mu \bar{\lambda}_{h}\right)\right\|_{L^{\infty}\left(\Omega_{h}\right)} .
$$

With these observations the proof of Theorem 4.5 is immediate. Finally, we have the following error estimates.

TheOrem 5.1. Let $\bar{u}$ be a local minimum of problem $(\mathrm{P})$ and let $\left\{\bar{u}_{h}\right\}_{h>0}$ be a sequence of local minima of problems $\left(\mathrm{Q}_{h}\right)$ such that $\left\|\bar{u}-\bar{u}_{h}\right\|_{L^{\infty}\left(\Omega_{h}\right)} \rightarrow 0$. Let us assume that (4.15) holds. Then there exists a constant $C>0$ independent of $h$ such that

$$
\begin{aligned}
& \left\|\bar{u}-\bar{u}_{h}\right\|_{L^{2}\left(\Omega_{h}\right)}+\left\|\bar{\lambda}-\bar{\lambda}_{h}\right\|_{L^{2}\left(\Omega_{h}\right)} \leq C h^{2}, \\
& \left\|\bar{u}-\bar{u}_{h}\right\|_{L^{\infty}\left(\Omega_{h}\right)}+\left\|\bar{\lambda}-\bar{\lambda}_{h}\right\|_{L^{\infty}\left(\Omega_{h}\right)} \leq C h^{2-\frac{n}{\bar{p}}}|\log h|, \\
& \left\|\bar{y}-\bar{y}_{h}\right\|_{L^{2}\left(\Omega_{h}\right)}+\left\|\bar{\varphi}-\bar{\varphi}_{h}\right\|_{L^{2}\left(\Omega_{h}\right)} \\
& +h\left(\left\|\bar{y}-\bar{y}_{h}\right\|_{H^{1}\left(\Omega_{h}\right)}+\left\|\bar{\varphi}-\bar{\varphi}_{h}\right\|_{H^{1}\left(\Omega_{h}\right)}\right) \leq C h^{2}, \\
& \left\|\bar{y}-\bar{y}_{h}\right\|_{L^{\infty}\left(\Omega_{h}\right)}+\left\|\bar{\varphi}-\bar{\varphi}_{h}\right\|_{L^{\infty}\left(\Omega_{h}\right)} \leq C h^{2-\frac{n}{\bar{p}}}|\log h| .
\end{aligned}
$$

Proof. Arguing as in the previous section we see that the inequality (4.14) is valid for any $u_{h} \in \mathbb{K}$. Then we select $u_{h}=\bar{u}$ and (4.14) becomes

$$
\left[F^{\prime}\left(\bar{u}_{h}\right)-F^{\prime}(\bar{u})\right]\left(\bar{u}_{h}-\bar{u}\right) \leq\left[F_{h}^{\prime}\left(\bar{u}_{h}\right)-F^{\prime}\left(\bar{u}_{h}\right)\right]\left(\bar{u}-\bar{u}_{h}\right)+\mu \int_{\Omega}\left(\bar{\lambda}-\bar{\lambda}_{h}\right)\left(\bar{u}_{h}-\bar{u}\right) \mathrm{d} x .
$$

Obviously Lemmas $4.6,4.7$, and 4.9 are still valid; then applied to the previous inequality we obtain the estimate $\left\|\bar{u}-\bar{u}_{h}\right\|_{L^{2}\left(\Omega_{h}\right)} \leq C h^{2}$. This estimate combined with (4.7a) and (4.7b) proves (5.2c). Now (5.2d) is an immediate consequence of (4.7c) and the estimate obtained for the controls. The $L^{\infty}\left(\Omega_{h}\right)$ estimate for the controls follows from $(5.2 \mathrm{~d})$ and the representation formulas $(3.5 \mathrm{a})$ and (5.1a). Finally, the estimates for $\bar{\lambda}-\bar{\lambda}_{h}$ are consequences of the estimates (5.2c) and (5.2d) and the representation formulas (3.5c) and (5.1c).

6. Numerical validation. The purpose of the numerical examples is to verify the theoretical results of the full discretization (section 4) and variational discretization approaches (section 5). Let us fix the parameters of problem (P). The domain $\Omega=B_{1}(0) \subset \mathbb{R}^{2}$ is the unit circle, and the state equation (2.1) is given by

$$
\begin{aligned}
-\Delta y+y^{3}=u & \text { in } \Omega, \\
y=0 & \text { on } \Gamma .
\end{aligned}
$$

Copyright $\odot$ by SIAM. Unauthorized reproduction of this article is prohibited. 
The part $L$ of the objective is the standard tracking type functional, i.e., $L(x, y)=$ $\frac{1}{2}\left(y-y_{d}(x)\right)^{2}$ with $y_{d}\left(x_{1}, x_{2}\right)=4 \sin \left(2 \pi x_{1}\right) \sin \left(\pi x_{2}\right) \mathrm{e}^{x_{1}}$. For the remaining parameters we choose

$$
\begin{aligned}
& \nu=2 \cdot 10^{-3}, \quad \alpha=-12 \\
& \mu=3 \cdot 10^{-2}, \quad \beta=12 .
\end{aligned}
$$

The adjoint equation (3.4b) is now given by

$$
\begin{aligned}
-\Delta \bar{\varphi}+3 \bar{y}^{2} \bar{\varphi} & =\bar{y}-y_{d} & & \text { in } \Omega, \\
\bar{\varphi} & =0 & & \text { on } \Gamma .
\end{aligned}
$$

The FEM library FEniCS $[10,14]$ and the Computational Geometry Algorithms Library (CGAL) [7] were used for all discretization related aspects of the implementation.

6.1. Full discretization. As described above, we employ continuous piecewise linear $\left(\mathcal{P}_{1}\right)$ discretizations of the state $y$ and adjoint state $\varphi$ and a piecewise constant approximation $\left(\mathcal{P}_{0}\right)$ of the control $u$. Now, the discretized optimality conditions for $\left(\mathrm{P}_{h}\right)$ are as follows:

$$
\begin{aligned}
& \text { Find }\left(y_{h}, \varphi_{h}, u_{h}\right) \in Y_{h} \times Y_{h} \times \mathcal{U}_{h} \text { such that } \\
& \begin{cases}\int_{\Omega_{h}} \nabla y_{h} \cdot \nabla z_{h}+y_{h}^{3} z_{h} \mathrm{~d} x=\int_{\Omega_{h}} u_{h} z_{h} \mathrm{~d} x & \forall z_{h} \in Y_{h}, \\
\int_{\Omega_{h}} \nabla \varphi_{h} \cdot \nabla z_{h}+3 y_{h}^{2} \varphi_{h} z_{h} \mathrm{~d} x=\int_{\Omega_{h}}\left(y_{h}-y_{d}\right) z_{h} \mathrm{~d} x & \forall z_{h} \in Y_{h}, \\
u_{T}=\operatorname{Proj}_{[\alpha, \beta]}\left(-\frac{1}{\nu}\left[\varphi_{T}-\operatorname{Proj}_{[-\mu, \mu]}\left(\varphi_{T}\right)\right]\right) & \text { on all } T \in \mathcal{T}_{h},\end{cases}
\end{aligned}
$$

where $\varphi_{T}=\frac{1}{|T|} \int_{T} \varphi_{h} \mathrm{~d} x$ is the mean value of $\varphi_{h}$ on $T$. Note that we have inserted (4.4c) into (4.4a). The nonlinear system (6.1) is solved via a semismooth Newton method and for a sequence of different meshes, similar to [17]. An example for the discretized optimal control on two different meshes is displayed in Figure 6.1. The error with respect to the solution on the finest grid $\left(h^{*}=2^{-8}\right)$ is shown in Table 6.1. It confirms the linear rate of convergence w.r.t. $h$. The error in the $L^{\infty}$ norm falls below $12=\beta-0=0-\alpha$ only as soon as the relatively small margin between the parts of the domain where $\bar{u} \in\{\alpha, 0, \beta\}$ is resolved by the mesh. Note also that the meshes for this example are not nested.

We remark that the analysis above carries over to problems defined on a convex polygonal domain $\Omega$; see Remark 2.4. Indeed, we observe the same convergence rates for the case $\Omega=(0,1)^{2} \subset \mathbb{R}^{2}$ with all other problem data unchanged; see Table 6.1. The finest grid in this case was $h^{*}=2^{-9}$.

6.2. Variational discretization. As described above, we employ continuous piecewise linear $\left(\mathcal{P}_{1}\right)$ discretizations of the state $y$ and adjoint state $\varphi$ and a variational approximation of the control $u$, i.e., $\mathcal{U}_{h}=L^{\infty}(\Omega)$. Now, the discretized optimality 

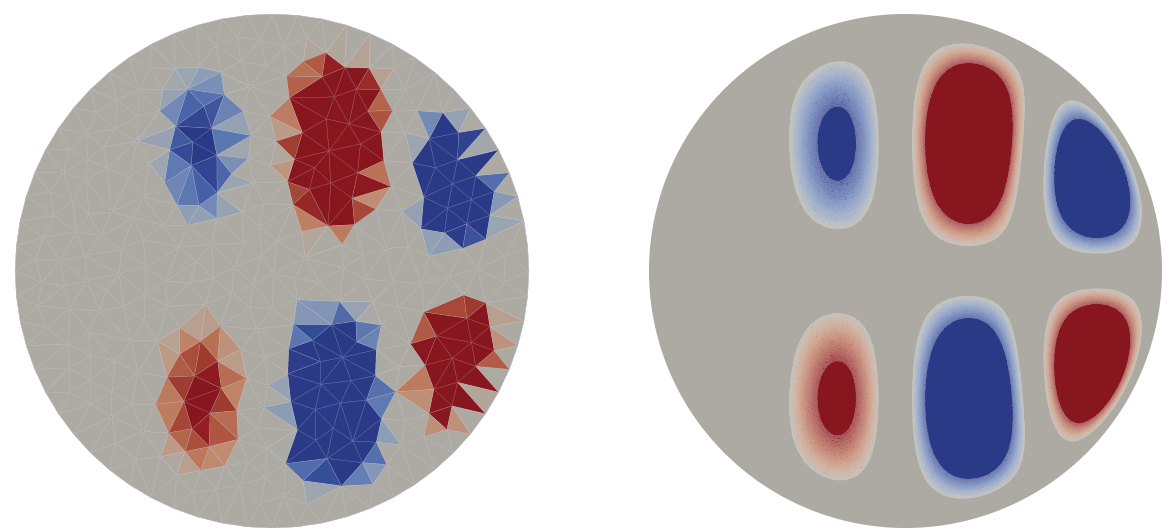

FIG. 6.1. Optimal control $\bar{u}_{h}$ for the case of full discretization on the unit circle, $h=2^{-3}$, and $h=2^{-8}$. Dark blue and dark red areas correspond to $\bar{u}_{h}= \pm 12$ and gray areas to $\bar{u}_{h}=0$. (References to color apply to online version only.)

TABLE 6.1

$L^{2}$ and $L^{\infty}$ errors in the control on the unit circle and the unit square in case of full discretization. The error was computed against the solution on the finest grid, using $h^{*}=2^{-8}$ on the unit circle and $h^{*}=2^{-9}$ on the unit square.

\begin{tabular}{ccclc}
\hline \multicolumn{3}{c}{ Unit circle } & \multicolumn{2}{c}{ Unit square } \\
$h$ & $\left\|\bar{u}_{h}-\bar{u}_{h^{*}}\right\|_{L^{2}}$ & $\left\|\bar{u}_{h}-\bar{u}_{h^{*}}\right\|_{L^{\infty}}$ & $\left\|\bar{u}_{h}-\bar{u}_{h^{*}}\right\|_{L^{2}}$ & $\left\|\bar{u}_{h}-\bar{u}_{h^{*}}\right\|_{L^{\infty}}$ \\
\hline $2^{-1}$ & 10.8288 & 14.8802 & 8.3472 & 12.0000 \\
$2^{-2}$ & 6.9220 & 12.0000 & 5.8162 & 12.0000 \\
$2^{-3}$ & 3.5205 & 12.0000 & 2.8185 & 12.0000 \\
$2^{-4}$ & 1.6310 & 12.0000 & 1.5246 & 12.0000 \\
$2^{-5}$ & 0.8463 & 8.3641 & 0.7899 & 9.9094 \\
$2^{-6}$ & 0.4300 & 5.1796 & 0.3983 & 5.0352 \\
$2^{-7}$ & 0.2360 & 2.9225 & 0.1989 & 2.1658 \\
$2^{-8}$ & & & 0.0896 & 0.7267 \\
\hline \hline
\end{tabular}

conditions for $\left(\mathrm{Q}_{h}\right)$ are as follows:

(6.2) Find $\left(y_{h}, \varphi_{h}\right) \in Y_{h} \times Y_{h}$ such that

$$
\begin{cases}\int_{\Omega_{h}} \nabla y_{h} \cdot \nabla z_{h}+y_{h}^{3} z_{h} \mathrm{~d} x=\int_{\Omega_{h}} u_{h}\left(\varphi_{h}\right) z_{h} \mathrm{~d} x & \forall z_{h} \in Y_{h}, \\ \int_{\Omega_{h}} \nabla \varphi_{h} \cdot \nabla z_{h}+3 y_{h}^{2} \varphi_{h} z_{h} \mathrm{~d} x=\int_{\Omega_{h}}\left(y_{h}-y_{d}\right) z_{h} \mathrm{~d} x & \forall z_{h} \in Y_{h},\end{cases}
$$

where we use the relationship between $u_{h}$ and $\varphi_{h}$

$$
u_{h}\left(\varphi_{h}\right)=\operatorname{Proj}_{[\alpha, 0]}\left(\frac{1}{\nu}\left(-\varphi_{h}+\mu\right)\right)+\operatorname{Proj}_{[0, \beta]}\left(\frac{1}{\nu}\left(-\varphi_{h}-\mu\right)\right),
$$

due to (5.1). The nonlinear system (6.2) is solved via a semismooth Newton method and for a sequence of different meshes.

The error in $\varphi$ with respect to the solution on the finest grid $\left(h^{*}=2^{-8}\right)$ for the unit circle is shown in Table 6.2. It confirms the quadratic rate of convergence w.r.t. $h$. By the Lipschitz continuity of the projection (6.3) with Lipschitz constant $1 / \nu$, 
the same convergence order holds for the control $\bar{u}_{h}$. Since the computed controls $u_{h}$ have kinks on the triangles of the mesh and since the meshes are not nested, computing the actual error in $u_{h}$ would be rather complicated. Table 6.2 shows the results for the unit square with a finest grid size $h^{*}=2^{-9}$.

TABLE 6.2

$L^{2}$ and $L^{\infty}$ errors in the adjoint state on the unit circle and unit square in case of variational discretization.

\begin{tabular}{cllll}
\hline & \multicolumn{2}{c}{ Unit circle } & \multicolumn{2}{c}{ Unit square } \\
Mesh size $h$ & $\left\|\bar{\varphi}_{h}-\bar{\varphi}_{h^{*}}\right\|_{L^{2}}$ & $\left\|\bar{\varphi}_{h}-\bar{\varphi}_{h^{*}}\right\|_{L^{\infty}}$ & $\left\|\bar{\varphi}_{h}-\bar{\varphi}_{h^{*}}\right\|_{L^{2}}$ & $\left\|\bar{\varphi}_{h}-\bar{\varphi}_{h^{*}}\right\|_{L^{\infty}}$ \\
\hline $2^{-1}$ & 0.041365 & 0.125195 & 0.049961 & 0.175814 \\
$2^{-2}$ & 0.015961 & 0.053100 & 0.021283 & 0.072157 \\
$2^{-3}$ & 0.005115 & 0.021832 & 0.006799 & 0.026508 \\
$2^{-4}$ & 0.001160 & 0.004785 & 0.001816 & 0.007255 \\
$2^{-5}$ & 0.000287 & 0.001297 & 0.000461 & 0.001843 \\
$2^{-6}$ & 0.000068 & 0.000372 & 0.000114 & 0.000461 \\
$2^{-7}$ & 0.000014 & 0.000092 & 0.000027 & 0.000113 \\
$2^{-8}$ & & & 0.000006 & 0.000026 \\
\hline \hline
\end{tabular}

\section{REFERENCES}

[1] N. Arada, E. CASAS, ANd F. TröLtzsch, Error estimates for the numerical approximation of a semilinear elliptic control problem, Comput. Optim. Appl., 23 (2002), pp. 201-229.

[2] F. Bonnans and A. Shapiro, Perturbation Analysis of Optimization Problems, SpringerVerlag, New York, 2000.

[3] S. Brenner and L. Scott, The Mathematical Theory of Finite Element Methods, SpringerVerlag, New York, 1994.

[4] E. CASAS, Boundary control of semilinear elliptic equations with pointwise state constraints, SIAM J. Control Optim., 31 (1993), pp. 993-1006.

[5] E. Casas and M. Mateos, Uniform convergence of the FEM. Applications to state constrained control problems, Comput. Appl. Math., 21 (2002), pp. 67-100.

[6] E. Casas, M. Mateos, and F. Tröltzsch, Error estimates for the numerical approximation of boundary semilinear elliptic control problems, Comput. Optim. Appl., 31 (2005), pp. 193219.

[7] CGAL, Computational Geometry Algorithms Library, http://www.cgal.org.

[8] F. Clarke, Optimization and Nonsmooth Analysis, John Wiley \& Sons, New York, 1983.

[9] C. Clason And K. Kunisch, A duality-based approach to elliptic control problems in nonreflexive Banach spaces, ESAIM Control Optim. Calc. Var., 17 (2011), pp. 243-266.

[10] FEniCS, FEniCS Project, http://www.fenicsproject.org/ (2007).

[11] P. Grisvard, Elliptic Problems in Nonsmooth Domains, Pitman, Boston, 1985.

[12] E. Hewitt and K. Stromberg, Real and Abstract Analysis, Springer-Verlag, New York, 1965.

[13] M. Hinze, A variational discretization concept in control constrained optimization: The linearquadratic case, Comput. Optim. Appl., 30 (2005), pp. 45-61.

[14] A. LogG, Automating the finite element method, Arch. Comput. Methods Eng., 14 (2007), pp. 93-138.

[15] P. Raviart and J. Thomas, Introduction à L'analyse Numérique des Equations aux Dérivées Partielles, Masson, Paris, 1983.

[16] A. Schatz, Pointwise error estimates and asymptotic error expansion inequalities for the finite element method on irregular grids: Part I. Global estimates, Math. Comp., 67 (1998), pp. 877-899.

[17] G. STADLER, Elliptic optimal control problems with $L^{1}$-control cost and applications for the placement of control devices, Comput. Optim. Appl., 44 (2009), pp. 159-181.

[18] F. Tröltzsch, Optimal Control of Partial Differential Equations, Grad. Stud. Math. 112, American Mathematical Society, Providence, RI, 2010.

[19] G. Wachsmuth And D. WaChSmuth, Convergence and regularization results for optimal control problems with sparsity functional, ESAIM Control Optim. Calc. Var., 17 (2011), pp. 858-866. 